doi:10.13108/2020-12-1-56

\title{
ON PRESERVATION OF GLOBAL SOLVABILITY OF CONTROLLED SECOND KIND OPERATOR EQUATION
}

\author{
A.V. CHERNOV
}

\begin{abstract}
For a controlled evolution second kind operator equation in a Banach space considered on a finite time segment, we obtain sufficient conditions for the preservation of global solvability under small (with respect to the right-hand side increment with a fixed state) control variations. In addition, we establish an estimate for the global solution increment under a control variation and conditions for uniqueness of the solution corresponding to an arbitrary fixed control. Most essential differences from former results on the preservation of global solvability of controlled distributed systems are as follows. A solution to the abstract equation representing an evolution controlled distributed system can be sought in arbitrary space $W[0 ; T]$ of time functions with values in a Banach space $X$ and not necessarily in the space of continuous functions with values in $X$ or in a Lebesgue space. An estimate for the solution increment under a control variation is also obtained with respect to the norm of the space $W[0 ; T]$. Moreover, the right hand sides of the partial differential equations associated with a controlled distributed system may include not only the function of state but also its generalized derivatives. As examples, we study the preservation of global solvability for the nonlinear Navier-Stokes system, the Benjamin-Bona-Mahony-Burgers equation, and also for certain strongly nonlinear pseudo-parabolic equations.
\end{abstract}

Keywords: nonlinear evolution operator equation of second kind in a Banach space, preservation of global solvability, nonlinear Navier-Stokes system, Benjamin-Bona-Mahony-Burgers equation, strongly nonlinear pseudo-parabolic equations.

Mathematics Subject Classification: 47J05, 47J35, 47N10

\section{INTRODUCTION}

Let $X$ be a Banach space. Following [1, Ch. IV, Sect. 1], we shall employ the following notations for Banach spaces of functions defined on the segment $[0 ; T]$ with values in the space $X$.

$C^{k}([0 ; T] ; X)$ for $k=0,1, \ldots$ is the set of all functions $\varphi:[0 ; T] \rightarrow X$ possessing continuous derivatives up to order $k$ equipped with the norm $\|\varphi\|_{C^{k}([0 ; T] ; X)}=\sum_{j=0}^{k} \max _{t \in[0 ; T]}\left\|\varphi^{(j)}(t)\right\|_{X}$.

$L_{q}([0 ; T] ; X)$ for $q \in[1 ; \infty)$ is the set of all functions $\varphi:[0 ; T] \rightarrow X$ measurable in the Bochner sense, for which the integral $\int_{0}^{T}\|\varphi(t)\|_{X}^{q} d t$ is finite; for the measurability in the Bochner sense see, for instance, [1, Ch. IV, Sect. 1]. The norm is defined as follows:

$$
\|\varphi\|_{L_{q}([0 ; T] ; X)}=\left(\int_{0}^{T}\|\varphi(t)\|_{X}^{q} d t\right)^{\frac{1}{q}} .
$$

A.V. Chernov, On preservation of GLObal SOlvability of CONTROLled SECOND Kind operator EQUATION.

(C) Chernov A.V. 2020.

Submitted August 27, 2019. 
$L_{\infty}([0 ; T] ; X)$ is the set of all essentially bounded functions $\varphi:[0 ; T] \rightarrow X$ measurable in the Bochner sense. The norm is introduced as $\|\varphi\|_{L_{\infty}([0 ; T] ; X)}=\operatorname{vraisup}_{t \in[0 ; T]}\|\varphi(t)\|_{X}$.

We also adopt the following convention. In a phrase like $\langle v \in V$ is an object», the word «object» concerns each element $v$ in a set $V$.

In the present paper we consider a controlled evolution operator equation of second kind in the Banach space $X$

$$
\varphi=\mathcal{F}[f[u](\varphi)], \quad \varphi \in W[0 ; T] \subset L_{q}([0 ; T] ; X),
$$

on a finite time interval $[0 ; T]$, where $u$ is control. For this equation we obtain conditions ensuring stability of existence of global solutions (SEGS). In other words, these conditions ensure preservation of global solvability under variations of control small with respect to a variation of the right hand side for a fixed state. In particular, given an operator differential equations in the Banach space $X$ like in [1, Ch. V, Sect. 1], with a fixed right hand side $f \in L_{2}([0 ; T] ; X)$, then as the operator $\mathcal{F}$, a mapping serves, which maps the right hand side $f$ into the solution $\varphi$ of this equation for a fixed initial condition. If we consider a similar operator differential equation with a right hand side depending on a phase variable and control, then we can represent it as the studied operator equation.

SEGS issue is topical in obtaining necessary conditions of optimality in problems of optimal control, calculating the gradients of functionals in such problems and justifying corresponding numerical optimization methods. If there is no information about SEGS while varying the optimal control, in particular, in obtaining necessary conditions of optimality, one usually proceeds to considering the pairs «control-state», see, for instance, [2], [3, Ch. 2]. As a result, one has to treat the state equation as a phase restriction of a special type. This gives rise to certain technical difficulties; we can mention, for instance, an adapted penalty method proposed in [2].

In [2], a series of unsolved problems was formulated, namely, a series of controlled distributed systems was described, for which we failed to obtain necessary optimality conditions by means of the adapted penalty method. Meanwhile, in [4, Ch. 5, Sect. 2, Subsect. 2], 5. Ch. 3, Sect. 1], some problems from this series were presented, in which the necessary optimality conditions were obtained by employing SEGS theory. The matter is that if an information on SEGS is present, one can employ an alternative approach based on considering the functionals in the optimization problem as functions depending on control only. While studying here various issues, corresponding theorems and their generalizations in the functional analysis are to be used, see, for instance, [6], [7]. In particular, one can employ the technique of control parametrization for distributed control systems, see $[7 \mid$. Thus, the presence of SEGS gives additional opportunities for obtaining necessary optimality conditions in optimal control problems and their numerical solving.

We observe that the fail of the global solvability of an evolution controlled system associated with a differential or integro-differential equation is very likely, when the growth order of the right hand side in the corresponding equation with respect to the phase variable exceeds the linear growth, see demonstrative examples in [6], [8], [9, Introduction, Sect. 2]. Once the differential operator involves a nonlinearity, the situation becomes worse, see, for instance, [10], 11.

In studying various control problems, apart of a simple postulating the global solvability of a controlled problem for all admissible controls, various researchers employ usually some general or specific results on global solvability based on Browder-Minty theorem, Lax-Milgram theorem, Schauder theorem and others, see, for instance, [12]. These results are usually known in the theory of differential or integro-differential equations of specific form with a non-controlled right hand side depending nonlinearly on the state variable. A vast literature is devoted to the global solvability conditions of equations with a non-controlled right hand side, see, for 
instance, [13], |14], |15], |16], |17], |18|, |19], |20], |21], |22], |23], |24], |25]. At the same time, while studying the global solvability of initial-boundary problems with a nonlinear right hand side depending on the control, it makes sense to employ the information on the presence and nature of this dependence. In particular, it can turn out that the presence or absence of the global solvability depends essentially on how large the controlled parameters involved in the nonlinear right hand side of the equation are varied. In many situations one succeeds to prove that if, for instance, a system is globally solvable for some fixed control, then it keeps this property for all sufficiently small in a proper sense variations of this control; at the same time, for some admissible controls there can be no global solvability. Exactly this property accompanied by the uniqueness of the solution is called the stability of existence of global solutions or, more generally, the preservation of unique global solvability, see, for instance, the surveys in [26], [27], [28], [29].

Earlier, in studying the SEGS for controlled distributed systems, there was employed a method based on reducing these systems to a Volterra functional-operator (operator) equation in a Lebesgue (or, more generally, in a Banach ideal) space of measurable functions. Then corresponding abstract results were applied. More details on the history of developing the method of Volterra operator for obtaining SEGS conditions for controlled distributed systems can be found in the above cited surveys [26], [27], [28], [29]. In work [29], there was obtained an SEGS test for initial-boundary value problem related with a controlled semi-linear equation of a global electric circuit. Here the same idea on reducing to a Volterra functional-operator equation was employed; the equation was of Hammerstein type. A principle difference from former results was that the problem did not admit a reduction to an abstract equation in a Banach ideal space. The equation was considered in a space $C([0, T] ; X)$ with some Banach space $X$ of functions defined on a domain $\Omega \subset \mathbb{R}^{n}$. At the same time, the technique of [29] can be applied to semi-linear equations only. In [30], there was proved a condition for total (over all admissible controls) preservation of global solvability of evolution operator equation of first kind of general form with a controlled additional nonlinearity in a Banach space. The technique of [30] can be applied also to essentially nonlinear partial differential equations of evolution type. The matter of the condition in $|30|$ is the total preservation of global solvability (TPGS) of the mentioned equation was guaranteed by assuming the global solvability for some majorizing uniformly in all controls integral equation for an unknown function depending on the time variable only. At that, the majorizing equation was constructed on the base of postulated estimates for the controlled additional nonlinearity.

The present paper is a result of an essential revisiting of works [29|, |30|. First, the results of [29], [30] can be reformulated as conditions for SEGS and TPGS, respectively, for an evolution operator equation of second kind and in fact, such approach is more natural. Second, the space $C([0 ; T] ; X)$ serving in the cited works as the space, in which the solution is sought, can be poorly adapted for satisfying made assumptions. Third, the results of [29], |30| allow one to estimate the solution only in the norm of the space $C([0 ; T] ; X)$. At the same time, in applications, one can need to estimate in norm of the space $W[0 ; T]$ (say, Sobolev space) and to seek the solution in this space. Fourth, assumed apriori pointwise (as $t \in[0 ; T]$ ) estimates for the solution of the corresponding equation with a fixed right hand side independent on the phase variable, can be realized not as an integral with a varying upper limit as it was assumed but only as estimates in the norm of $W[0 ; T]$. Fifth, in [30], the right hand side of the equation could not involve the generalized derivatives of the state function, but only this function itself. Exactly improving of the technique [29], [30] according the above remarks is the main result of the present paper. As examples we consider a controlled nonlinear non-stationary system of Navier-Stokes equations, Benjamin-Bona-Mahony-Burgers equation, as well as some strongly nonlinear pseudo-parabolic equations. The results of works [29], [30] here are not applicable in view of the above remarks, while this is the case the results of the present paper. 
Nevertheless, as in [29], [30], the theorem on preservation the global solvability is proved by successive continuation of the solution along the time scale as earlier there was used a continuation along the Volterra chain of the operator in the right hand side of Hammerstein type equation representing the studied controlled system. The uniqueness of the solution is proved by a similar continuation of the difference of two assumed solutions corresponding to the same control.

\section{Formulation OF MAIN RESUlts}

Let $X, Y$ be real Banach spaces, $T>0, W_{0}[0 ; T]$ be a closed set in the Banach space $W[0 ; T]$, whose elements are the functions with values in $X$; in particular, the embedding $W[0 ; T] \subset L_{q}([0 ; T] ; X)$ can hold. More precisely, we assume that a scale of Banach spaces $W[0 ; \tau]$ is given and respectively, that of closed subsets $W_{0}[0 ; \tau] \subset W[0 ; \tau], \tau \in(0 ; T]$. In view of practical applications, $W_{0}[0 ; T]$ is a set of functions in $W[0 ; T]$ satisfying some initial condition (initial-boundary conditions) written in some given form.

Let $\mathcal{B}([0 ; T] ; Y)$ be some linear normed space of measurable in the Bochner sense functions $t \in[0 ; T] \rightarrow z(t) \in Y, \mathcal{B}([\tau ; \xi] ; Y)$ be the corresponding induced space of restrictions on $[\tau ; \xi]$, $0 \leqslant \tau<\xi \leqslant T$, and the following natural assumptions hold true:

$$
\begin{array}{lll}
\|z\|_{\mathcal{B}([\tau ; \xi] ; Y)} \leqslant\|z\|_{\mathcal{B}([0 ; T] ; Y)}, & \|z\|_{\mathcal{B}([0 ; \xi] ; Y)} \leqslant\|z\|_{\mathcal{B}([0 ; \tau] ; Y)}+\|z\|_{\mathcal{B}([\tau ; \xi] ; Y)}, \quad \tau>0 \\
\|z(\cdot)\|_{Y} \in \mathcal{B}[\tau ; \xi]=\mathcal{B}([\tau ; \xi] ; \mathbb{R}), & \|z\|_{\mathcal{B}([\tau ; \xi] ; Y)}=\|\| z\left\|_{Y}\right\|_{\mathcal{B}[\tau ; \xi]},
\end{array}
$$

and the sum and the multiplication by a scalar are defined pointwise. In particular, as $\mathcal{B}[0 ; T]$, the spaces $C[0 ; T], L_{p}[0 ; T], p \in[1 ;+\infty]$ can serve. We assume that we are given a family of the operators $\mathcal{F}_{\tau}: \mathcal{B}([0 ; \tau] ; Y) \rightarrow W_{0}[0 ; \tau], \tau \in(0 ; T], \mathcal{F}=\mathcal{F}_{T}$, satisfying the following conditions:

$\mathbf{G}_{1}$ ) (Volterra property). For all $\tau, \xi \in(0 ; T], \tau \leqslant \xi, z_{\tau} \in \mathcal{B}([0 ; \tau] ; Y), z_{\xi} \in \mathcal{B}([0 ; \xi] ; Y)$, and corresponding images $\varphi_{\tau}=\mathcal{F}_{\tau} z_{\tau}, \varphi_{\xi}=\mathcal{F}_{\xi} z_{\xi}$, the condition $z_{\xi}(t)=z_{\tau}(t)$ for almost each $t \in[0 ; \tau]$, implies the identity $\left.\varphi_{\xi}\right|_{[0 ; \tau]}=\varphi_{\tau}$ in the space $W[0 ; \tau]$.

Hence, for each $z \in \mathcal{B}([0 ; T] ; Y)$, the equation of form

$$
\varphi=\mathcal{F}[z]
$$

possesses a unique local solution $\varphi_{\tau} \in W_{0}[0 ; \tau]$, which is determined as $\varphi_{\tau}=\mathcal{F}_{\tau}\left[\left.z\right|_{[0 ; \tau]}\right]$. This property allows us to construct the solution by continuing it from a small segment to a bigger one.

$\left.\mathbf{G}_{2}\right)$ For all $\tau, \xi \in[0 ; T], \tau<\xi, z_{j} \in \mathcal{B}([0 ; \xi] ; Y),\left.z_{1}\right|_{[0 ; \tau]}=\left.z_{2}\right|_{[0 ; \tau]}$ in the case $\tau>0$, $\left\|z_{j}\right\|_{\mathcal{B}([0 ; \xi] ; Y)} \leqslant \omega$, and corresponding $\varphi_{j}=\mathcal{F}_{\xi}\left[z_{j}\right], j=1,2$, the inequality

$$
\left\|\varphi_{1}-\varphi_{2}\right\|_{W[0 ; \xi]} \leqslant \mathcal{N}(\omega) \alpha_{0}(\xi-\tau)\left\|z_{1}-z_{2}\right\|_{\mathcal{B}([\tau ; \xi] ; Y)}
$$

holds, where $\mathcal{N}: \mathbb{R}_{+} \rightarrow \mathbb{R}_{+}$is a non-decreasing function, $\alpha_{0}: \mathbb{R}_{+} \rightarrow \mathbb{R}_{+}$is a continuous function, and $\alpha_{0}(\delta)>0$ as $0<\delta<\delta_{0}$.

$\left.\mathbf{G}_{3}\right) \sup _{\tau \in(0 ; T]}\left\|\mathcal{F}_{\tau}(0)\right\|_{W[0 ; \tau]}<+\infty$.

Now we add a dependence on the phase variable and on the control to the right hand side. Namely, we consider a controlled analogue of equation (2.1):

$$
\varphi=\mathcal{F}[f[u](\varphi)], \quad \varphi \in W[0 ; T] .
$$

Here $u \in U$ is a control, $U \subset \mathcal{U}$ is a given set in some, generally speaking arbitrary, space $\mathcal{U}$. We assume that for all $u \in U$ the operator $f[u]=f_{T}[u]$ is well-defined and this operator 
corresponds to the family of the operators $f_{\tau}[u]: W[0 ; \tau] \rightarrow \mathcal{B}([0 ; \tau] ; Y), \tau \in(0 ; T]$, possessing the following properties:

$\left.\mathbf{F}_{1}\right)$ For all $\tau, \xi \in(0 ; T], \tau \leqslant \xi$, and $\psi_{\xi} \in W[0 ; \xi],\left.\psi_{\xi}\right|_{[0 ; \tau]}=\psi_{\tau} \in W[0 ; \tau]$ the identity $f_{\xi}[u]\left(\psi_{\xi}\right)(t)=f_{\tau}[u]\left(\psi_{\tau}\right)(t)$ holds for almost each $t \in[0 ; \tau]$.

$\left.\mathbf{F}_{2}\right)$ For all $\tau, \xi \in(0 ; T], \tau \leqslant \xi, \psi_{1}, \psi_{2} \in W_{0}[0 ; \xi],\left\|\psi_{i}\right\|_{W[0 ; \xi]} \leqslant M, i=1,2$, the inequality

$$
\left\|f_{\xi}[u]\left(\psi_{1}\right)-f_{\xi}[u]\left(\psi_{2}\right)\right\|_{\mathcal{B}([\tau ; \xi] ; Y)} \leqslant \alpha_{1}(\xi-\tau) \beta_{1}(M)\left\|\psi_{1}-\psi_{2}\right\|_{W[0 ; \xi]}
$$

holds, where $\alpha_{1}: \mathbb{R}_{+} \rightarrow \mathbb{R}_{+}$is a continuous function, $\beta_{1}: \mathbb{R}_{+} \rightarrow \mathbb{R}_{+}$is a non-decreasing function.

$\left.\mathbf{F}_{3}\right)$ There exists a non-decreasing function $\beta_{0}: \mathbb{R}_{+} \rightarrow \mathbb{R}_{+}$such that for all

$$
\begin{aligned}
& u, \bar{u} \in U, \quad \tau, \xi \in(0 ; T], \quad \tau<\xi, \quad \gamma>0, \quad \varphi \in W_{0}[0 ; \tau], \\
& \bar{\varphi} \in W_{0}[0 ; \xi], \quad\|\varphi\|_{W[0 ; \tau]} \leqslant M, \quad\|\bar{\varphi}\|_{W[0 ; \xi]} \leqslant M,
\end{aligned}
$$

in the case

$$
\rho_{\tau, \xi}(u, \bar{u})=\left\|f_{\xi}[u](\bar{\varphi})-f_{\xi}[\bar{u}](\bar{\varphi})\right\|_{\mathcal{B}([\tau ; \xi] ; Y)} \leqslant \gamma
$$

there exists a function

$$
\widetilde{z}=Z[\tau, \xi, \gamma, u, \bar{u}, \varphi, \bar{\varphi}] \in \mathcal{B}([0 ; \xi] ; Y)
$$

such that

$$
\left.\widetilde{z}\right|_{[0 ; \tau]}=f_{\tau}[u](\varphi), \quad\left\|\widetilde{z}-f_{\xi}[\bar{u}](\bar{\varphi})\right\|_{\mathcal{B}([\tau ; \xi] ; Y)} \leqslant \beta_{0}(M)\left\{\gamma+\left\|f_{\tau}[u](\varphi)-f_{\xi}[u](\bar{\varphi})\right\|_{\mathcal{B}([0 ; \tau] ; Y)}\right\} .
$$

Remark 2.1. At first glance, condition $\mathbf{F}_{3}$ ) can seem to be too exotic and difficult to check. In fact, as we shall show below, it holds, for instance, for Lebesgue spaces $\mathcal{B}=L_{p}, p \in[1 ; \infty]$, and the space of continuous functions $\mathcal{B}=C$.

Lemma 2.1. Let the space $\mathcal{B}([0 ; \xi] ; Y)$ be such that for the characteristic function $\chi=\chi_{(\tau ; \xi]}$, for all $z_{1} \in \mathcal{B}([0 ; \tau] ; Y), z_{2} \in \mathcal{B}([0 ; \xi] ; Y)$ and for extension $z_{1, \xi}$ by zero of the function $z_{1}$ on $[0 ; \xi]$ we have $z_{1, \xi} \in \mathcal{B}([0 ; \xi] ; Y), \chi z_{2} \in \mathcal{B}([0 ; \xi] ; Y)$. This property obviously holds for the Lebesgue spaces $L_{p}([0 ; \xi] ; Y), p \in[1 ; \infty]$. Then condition $\left.\mathbf{F}_{3}\right)$ is satisfied.

Proof. It is sufficient to let

$$
\widetilde{z}(t)= \begin{cases}f_{\tau}[u](\varphi)(t), & t \in[0 ; \tau] \\ f_{\xi}[u](\bar{\varphi})(t), & t \in(\tau ; \xi]\end{cases}
$$

It is clear that $\widetilde{z}=z_{1, \xi}+\chi z_{2} \in \mathcal{B}([0 ; \xi] ; Y)$, where $z_{1}=f_{\tau}[u](\varphi), z_{2}=f_{\xi}[u](\bar{\varphi})$. At that,

$$
\left.\widetilde{z}\right|_{[0 ; \tau]}=z_{1}, \quad\left\|\widetilde{z}-f_{\xi}[\bar{u}](\bar{\varphi})\right\|_{\mathcal{B}([\tau ; \xi] ; Y)}=\left\|f_{\xi}[u](\bar{\varphi})-f_{\xi}[\bar{u}](\bar{\varphi})\right\|_{\mathcal{B}([\tau ; \xi] ; Y)} \leqslant \gamma .
$$

Lemma 2.2. Let $\mathcal{B}([0 ; \xi] ; Y)=C([0 ; \xi] ; Y)$. Then condition $\left.\mathbf{F}_{3}\right)$ is satisfied.

Proof. We denote

By assumptions,

$$
z=f_{\tau}[u](\varphi), \quad \widehat{z}=f_{\xi}[u](\bar{\varphi}), \quad \bar{z}=f_{\xi}[\bar{u}](\bar{\varphi})
$$

$$
\rho_{\tau, \xi}(u, \bar{u})=\|\widehat{z}-\bar{z}\|_{C([\tau ; \xi] ; Y)} \leqslant \gamma, \quad \gamma>0 .
$$

Since $\widehat{z} \in C([0 ; \xi] ; Y)$, there exists $\eta \in(\tau ; \xi)$ such that

$$
\|\widehat{z}(t)-\widehat{z}(\tau)\|_{Y} \leqslant \gamma \quad \text { for all } \quad t \in[\tau ; \eta] .
$$


Let $w:[\tau ; \xi] \rightarrow[0 ; 1] \subset \mathbb{R}$ be a continuous function such that $w(\tau)=1, w(t)=0$ for all $t \in[\eta ; \xi]$. We let

$$
\widetilde{z}(t)= \begin{cases}z(t), & t \in[0 ; \tau], \\ w(t) z(\tau)+(1-w(t)) \widehat{z}(t), & t \in[\tau ; \xi] .\end{cases}
$$

By construction, $\widetilde{z} \in C([0 ; \xi] ; Y)$. We have

$$
\|\widetilde{z}-\bar{z}\|_{C([\tau ; \xi] ; Y)} \leqslant\|\widetilde{z}-\widehat{z}\|_{C([\tau ; \xi] ; Y)}+\|\widehat{z}-\bar{z}\|_{C([\tau ; \xi] ; Y)} \leqslant\|\widetilde{z}-\widehat{z}\|_{C([\tau ; \eta] ; Y)}+\gamma
$$

since $\left.\widetilde{z}\right|_{[\eta ; \xi]}=\left.\widehat{z}\right|_{[\eta ; \xi]}$ for an arbitrary function $w(t)$. For an arbitrary $t \in[\tau ; \eta]$ we estimate

$$
\begin{aligned}
\|\widetilde{z}(t)-\widehat{z}(t)\|_{Y} & =w(t)\|z(\tau)-\widehat{z}(t)\|_{Y} \\
& \leqslant\|z(\tau)-\widehat{z}(\tau)\|_{Y}+\|\widehat{z}(\tau)-\widehat{z}(t)\|_{Y} \leqslant\|z-\widehat{z}\|_{C([0 ; \tau] ; Y)}+\gamma
\end{aligned}
$$

Hence, we obtain:

$$
\|\widetilde{z}-\bar{z}\|_{C([\tau ; \xi] ; Y)} \leqslant\|z-\widehat{z}\|_{C([0 ; \tau] ; Y)}+2 \gamma
$$

Theorem 2.1. Assume that Conditions $\left.\left.\left.\left.\mathbf{G}_{1}\right)-\mathbf{G}_{3}\right), \mathbf{F}_{1}\right), \mathbf{F}_{2}\right)$ and the identity $\alpha_{0}(0) \alpha_{1}(0)=0$ are satisfied. Then, for arbitrary $u \in U$, equation (2.2) can possess at most one solution.

Theorem 2.2. Assume that Conditions $\left.\left.\left.\left.\mathbf{G}_{1}\right)-\mathbf{G}_{3}\right), \mathbf{F}_{1}\right)-\mathbf{F}_{3}\right)$ and the identity $\alpha_{0}(0) \alpha_{1}(0)=$ 0 are satisfied. Assume also that a control $u=\bar{u} \in U$ gives rise to a global solution $\varphi=\bar{\varphi} \in$ $W[0 ; T]$ of equation (2.2). Then there exist numbers $\varepsilon>0$ and $C>0$ such that for each $u \in U$ obeying the estimate

$$
\rho(u, \bar{u})=\|f[u](\bar{\varphi})-f[\bar{u}](\bar{\varphi})\|_{\mathcal{B}([0 ; T] ; Y)} \leqslant \varepsilon
$$

there exists a unique global solution $\varphi \in W[0 ; T]$ of equation 2.2 and moreover,

$$
\|\varphi-\bar{\varphi}\|_{W[0 ; T]} \leqslant C \rho(u, \bar{u}) \text {. }
$$

We prove Theorems 2.1, 2.2 in Section 3.

\section{PROOF OF MAIN RESUlts}

For all $\tau, \xi \in(0 ; T], \tau \leqslant \xi, \varphi \in W_{0}[0 ; \tau]$ we define $\Phi[\xi, \varphi]$ as the set of all $\eta \in W_{0}[0 ; \xi]$ such that

$$
\inf _{\psi}\|\psi-\eta\|_{W[0 ; \xi]}=0
$$

where the infimum is taken over all $\psi \in W_{0}[0 ; \xi]$ such that $\left.\psi\right|_{[0 ; \tau]}=\varphi$ in the space $W[0 ; \tau]$.

Lemma 3.1. For all $\tau, \xi \in(0 ; T], \tau \leqslant \xi, \varphi \in W_{0}[0 ; \tau]$, the set $\Phi[\xi, \varphi]$ is closed in the space $W[0 ; \xi]$.

Proof. In the case $\Phi[\xi, \varphi]=\emptyset$ and also in the case of a finite set the statement is trivial. In what follows we assume that the set $\Phi[\xi, \varphi]$ is infinite.

Let $\left\{\eta_{m}\right\} \subset \Phi[\xi, \varphi],\left\|\eta_{m}-\eta_{0}\right\|_{W[0 ; \xi]} \rightarrow 0$ as $m \rightarrow \infty$. Since the set $W_{0}[0 ; \xi]$ is closed in the space $W[0 ; \xi]$, it is clear that $\eta_{0} \in W_{0}[0 ; \xi]$. We fix an arbitrary number $\varepsilon>0$ and we find a number $m_{\varepsilon} \in \mathbb{N}$ such that

$$
\left\|\eta_{m}-\eta_{0}\right\|_{W[0 ; \xi]}<\frac{\varepsilon}{2} \quad \text { for all } \quad m \geqslant m_{\varepsilon}
$$

According the definition of the set $\Phi[\xi, \varphi]$, there exists $\psi_{\varepsilon} \in W_{0}[0 ; \xi]$ such that

$$
\left.\psi_{\varepsilon}\right|_{[0 ; \tau]}=\varphi, \quad\left\|\psi_{\varepsilon}-\eta_{m_{\varepsilon}}\right\|_{W[0 ; \xi]}<\frac{\varepsilon}{2} .
$$


We estimate:

$$
\left\|\psi_{\varepsilon}-\eta_{0}\right\|_{W[0 ; \xi]} \leqslant\left\|\psi_{\varepsilon}-\eta_{m_{\varepsilon}}\right\|_{W[0 ; \xi]}+\left\|\eta_{m_{\varepsilon}}-\eta_{0}\right\|_{W[0 ; \xi]}<\varepsilon .
$$

Since the number $\varepsilon>0$ is arbitrary, this means that $\eta_{0} \in \Phi[\xi, \varphi]$.

Lemma 3.2. Assume that conditions $\left.\left.\mathbf{F}_{1}\right), \mathbf{F}_{2}\right)$ are satisfied. Then for all $\tau, \xi \in(0 ; T], \tau \leqslant \xi$, $\varphi \in W_{0}[0 ; \tau]$, and respectively, $\psi_{1}, \psi_{2} \in \Phi[\xi, \varphi], u \in U$, we have:

$$
\left\|f_{\xi}[u]\left(\psi_{1}\right)-f_{\xi}[u]\left(\psi_{2}\right)\right\|_{\mathcal{B}([0 ; \tau] ; Y)}=0 .
$$

Moreover, for $\varphi_{\xi} \in W_{0}[0 ; \xi],\left.\varphi_{\xi}\right|_{[0 ; \tau]}=\varphi_{\tau} \in W_{0}[0 ; \tau]$ we have

$$
\left\|f_{\xi}[u]\left(\psi_{s}\right)-f_{\xi}[u]\left(\varphi_{\xi}\right)\right\|_{\mathcal{B}([0 ; \tau] ; Y)} \leqslant\left\|f_{\tau}[u](\varphi)-f_{\tau}[u]\left(\varphi_{\tau}\right)\right\|_{\mathcal{B}([0 ; \tau] ; Y)}, \quad s=1,2 .
$$

Proof. 1. Let us prove formula (3.1). Let $\left\|\psi_{s}\right\|_{W[0 ; \xi]} \leqslant M, s=1,2$. We choose an arbitrary number $\varepsilon>0$, and according the definition of the set $\Phi[\xi, \varphi]$, we find $\widetilde{\psi}_{s} \in W_{0}[0 ; \xi]$ such that

$$
\left.\widetilde{\psi}_{s}\right|_{[0 ; \tau]}=\varphi, \quad \delta_{s}=\left\|\widetilde{\psi}_{s}-\psi_{s}\right\|_{W[0 ; \xi]} \leqslant 1, \quad \alpha_{1}(\xi) \beta_{1}(M+1) \delta_{s} \leqslant \frac{\varepsilon}{2}, \quad s=1,2 .
$$

It is clear that in this case $\left\|\widetilde{\psi}_{s}\right\|_{W[0 ; \xi]} \leqslant M+1, s=1,2$. Employing Conditions $\left.\mathbf{F}_{1}\right)$, $\mathbf{F}_{2}$ ), we get:

$$
\begin{aligned}
\left\|f_{\xi}[u]\left(\psi_{1}\right)-f_{\xi}[u]\left(\psi_{2}\right)\right\|_{\mathcal{B}([0 ; \tau] ; Y)} \leqslant & \left\|f_{\xi}[u]\left(\widetilde{\psi}_{1}\right)-f_{\xi}[u]\left(\widetilde{\psi}_{2}\right)\right\|_{\mathcal{B}([0 ; \tau] ; Y)} \\
& +\sum_{s=1}^{2}\left\|f_{\xi}[u]\left(\widetilde{\psi}_{s}\right)-f_{\xi}[u]\left(\psi_{s}\right)\right\|_{\mathcal{B}([0 ; \tau] ; Y)} \\
\leqslant & \left\|f_{\tau}[u](\varphi)-f_{\tau}[u](\varphi)\right\|_{\mathcal{B}([0 ; \tau] ; Y)}+\alpha_{1}(\xi) \beta_{1}(M+1) \sum_{s=1}^{2} \delta_{s} \leqslant \varepsilon .
\end{aligned}
$$

Since the number $\varepsilon$ is arbitrary, we obtain identity 3.1 .

2. Let $s \in \overline{1,2},\left\|\psi_{s}\right\|_{W[0 ; \xi]} \leqslant M,\left\|\varphi_{\xi}\right\|_{W[0 ; \xi]} \leqslant \bar{M}$. We are going to prove formula (3.2). We choose an arbitrary number $\varepsilon>0$ and according the definition of the set $\Phi[\xi, \varphi]$, we find $\widetilde{\psi}_{s} \in W_{0}[0 ; \xi]$ such that

$$
\left.\widetilde{\psi}_{s}\right|_{[0 ; \tau]}=\varphi, \quad \delta_{s}=\left\|\widetilde{\psi}_{s}-\psi_{s}\right\|_{W[0 ; \xi]} \leqslant 1, \quad \alpha_{1}(\xi) \beta_{1}(M+1) \delta_{s} \leqslant \varepsilon .
$$

It is clear that in this case $\left\|\widetilde{\psi}_{s}\right\|_{W[0 ; \xi]} \leqslant M+1$. Employing Conditions $\left.\mathbf{F}_{1}\right), \mathbf{F}_{2}$ ), we have:

$$
\begin{aligned}
\left\|f_{\xi}[u]\left(\psi_{s}\right)-f_{\xi}[u]\left(\varphi_{\xi}\right)\right\|_{\mathcal{B}([0 ; \tau] ; Y)} \leqslant & \left\|f_{\xi}[u]\left(\widetilde{\psi}_{s}\right)-f_{\xi}[u]\left(\psi_{s}\right)\right\|_{\mathcal{B}([0 ; \tau] ; Y)} \\
& +\left\|f_{\xi}[u]\left(\widetilde{\psi}_{s}\right)-f_{\xi}[u]\left(\varphi_{\xi}\right)\right\|_{\mathcal{B}([0 ; \tau] ; Y)} \\
\leqslant & \alpha_{1}(\xi) \beta_{1}(M+1) \delta_{s}+\left\|f_{\tau}[u](\varphi)-f_{\tau}[u]\left(\varphi_{\tau}\right)\right\|_{\mathcal{B}([0 ; \tau] ; Y)} \\
\leqslant & +\left\|f_{\tau}[u](\varphi)-f_{\tau}[u]\left(\varphi_{\tau}\right)\right\|_{\mathcal{B}([0 ; \tau] ; Y)} .
\end{aligned}
$$

Since the number $\varepsilon$ is arbitrary, we arrive at inequality.

In what follows, to avoid bulky notations, we shall omit the subscript $\tau$ in the notation $f_{\tau}[u]$ since its value is easily recovered from the context. 
Proof of Theorem 2.1. We argue by contrary assuming that a control $u \in U$ gives rise to two solutions $\varphi=\varphi_{1}$ and $\varphi=\varphi_{2}$ of equation (2.2). We denote

$$
\begin{aligned}
& z_{j}=f[u]\left(\varphi_{j}\right), \quad j=1,2, \quad \omega=\max _{j=1,2}\left\|z_{j}\right\|_{\mathcal{B}([0 ; T] ; Y)}, \quad \aleph_{s}=\max _{t \in[0 ; T]} \alpha_{s}(t), \quad s=0,1, \\
& \mathcal{N}_{\omega}=\mathcal{N}(\omega), \quad \gamma_{0}=\sup _{\tau \in(0 ; T]}\left\|\mathcal{F}_{\tau}(0)\right\|_{W[0 ; \tau]}, \quad M_{0}=\gamma_{0}+\mathcal{N}_{\omega} \omega \aleph_{0} .
\end{aligned}
$$

Here $\gamma_{0}<\infty$ by Condition $\left.\mathbf{G}_{3}\right)$. We observe that according Condition $\left.\mathbf{G}_{2}\right)$, for all $\tau \in(0 ; T]$ we have:

$$
\begin{aligned}
\left\|\left.\varphi_{j}\right|_{[0 ; \tau]}\right\|_{W[0 ; \tau]} & \leqslant\left\|\mathcal{F}_{\tau}(0)\right\|_{W[0 ; \tau]}+\left\|\mathcal{F}_{\tau}\left[\left.z_{j}\right|_{[0 ; \tau]}\right]-\mathcal{F}_{\tau}(0)\right\|_{W[0 ; \tau]} \\
& \leqslant \gamma_{0}+\mathcal{N}_{\omega} \aleph_{0}\left\|z_{j}\right\|_{\mathcal{B}([0 ; T] ; Y)} \leqslant M_{0}, \quad j=1,2 .
\end{aligned}
$$

By Conditions $\left.\mathbf{G}_{2}\right), \mathbf{F}_{2}$ ), the function $\alpha=\alpha_{0}(\cdot) \alpha_{1}(\cdot)$ is continuous and by the assumption of the theorem, it is such that $\alpha(0)=0$. This is why there exists a number $\delta \in\left(0 ; \delta_{0}\right)$ such that $\mathcal{N}_{\omega} \alpha(\delta) \beta_{1}\left(M_{0}\right) \leqslant \frac{1}{2}$. We choose an arbitrary partition of the segment $[0 ; T]$ of the form

$$
0=t_{0}<t_{1}<\ldots<t_{k}=T, \quad t_{i}-t_{i-1} \leqslant \delta, \quad i=\overline{1, k} .
$$

According Condition $\mathbf{G}_{1}$ ), we denote

$$
z_{j, i}=\left.z_{j}\right|_{\left[0 ; t_{i}\right]}, \quad \varphi_{j, i}=\left.\varphi_{j}\right|_{\left[0 ; t_{i}\right]} \in W\left[0 ; t_{i}\right] .
$$

We consider the segment $\left[0 ; t_{1}\right]$. According Condition $\left.\mathbf{G}_{1}\right)$, the identities $\varphi_{s, 1}=\mathcal{F}_{t_{1}}\left[z_{s, 1}\right]$, $s=1,2$, hold in the space $W\left[0 ; t_{1}\right]$. We are going to prove that $\varphi_{1,1}=\varphi_{2,1}$ in the space $W\left[0 ; t_{1}\right]$. We denote $\eta_{1}=\varphi_{1,1}-\varphi_{2,1}$. According Condition $\mathbf{G}_{2}$ ),

where by Conditions $\mathbf{F}_{1}$ ), $\mathbf{F}_{2}$ ),

$$
\left\|\eta_{1}\right\|_{W\left[0 ; t_{1}\right]} \leqslant \mathcal{N}_{\omega} \alpha_{0}(\delta)\left\|z_{1,1}-z_{2,1}\right\|_{\mathcal{B}\left(\left[0 ; t_{1}\right] ; Y\right)}
$$

$$
\begin{aligned}
\left\|z_{1,1}-z_{2,1}\right\|_{\mathcal{B}\left(\left[0 ; t_{1}\right] ; Y\right)} & =\left\|f[u]\left(\varphi_{1,1}\right)-f[u]\left(\varphi_{2,1}\right)\right\|_{\mathcal{B}\left(\left[0 ; t_{1}\right] ; Y\right)} \\
& \leqslant \alpha_{1}(\delta) \beta_{1}\left(M_{0}\right)\left\|\varphi_{1,1}-\varphi_{2,1}\right\|_{W\left[0 ; t_{1}\right]}
\end{aligned}
$$

Therefore,

$$
\left\|\eta_{1}\right\|_{W\left[0 ; t_{1}\right]} \leqslant \mathcal{N}_{\omega} \alpha(\delta) \beta_{1}\left(M_{0}\right)\left\|\eta_{1}\right\|_{W\left[0 ; t_{1}\right]} \leqslant \frac{1}{2}\left\|\eta_{1}\right\|_{W\left[0 ; t_{1}\right]}
$$

Thus,

$$
\frac{1}{2}\left\|\eta_{1}\right\|_{W\left[0 ; t_{1}\right]} \leqslant 0
$$

that is, $\eta_{1}=0$, or $\varphi_{1,1}=\varphi_{2,1}$ in the space $W\left[0 ; t_{1}\right]$.

Proceeding by induction, we assume that we have proved the identity $\varphi_{1, j-1}=\varphi_{2, j-1}$ in the space $W\left[0 ; t_{j-1}\right], j \in \overline{2, k}$. In view of this assumption, we are going to prove that $\varphi_{1, j}=\varphi_{2, j}$ in the space $W\left[0 ; t_{j}\right]$. According Condition $\left.\mathbf{G}_{1}\right)$, we have $\varphi_{s, j}=\mathcal{F}_{t_{j}}\left[z_{s, j}\right]$ in the space $W\left[0 ; t_{j}\right]$, $s=1,2$. We denote $\eta_{j}=\varphi_{1, j}-\varphi_{2, j}$. According the induction assumption and Condition $\mathbf{F}_{1}$ ), we have:

$$
\left\|z_{1, j}-z_{2, j}\right\|_{\mathcal{B}\left(\left[0 ; t_{j-1}\right] ; Y\right)}=\left\|f[u]\left(\varphi_{1, j-1}\right)-f[u]\left(\varphi_{2, j-1}\right)\right\|_{\mathcal{B}\left(\left[0 ; t_{j-1}\right] ; Y\right)}=0 .
$$

Then by Condition $\mathbf{G}_{2}$ ),

$$
\left\|\eta_{j}\right\|_{W\left[0 ; t_{j}\right]} \leqslant \mathcal{N}_{\omega} \alpha_{0}(\delta)\left\|z_{1, j}-z_{2, j}\right\|_{\mathcal{B}\left(\left[t_{j-1} ; t_{j}\right] ; Y\right)} .
$$

Here Condition $\mathbf{F}_{2}$ ) yields that

$$
\left\|z_{1, j}-z_{2, j}\right\|_{\mathcal{B}\left(\left[t_{j-1} ; t_{j}\right] ; Y\right)}=\left\|f[u]\left(\varphi_{1, j}\right)-f[u]\left(\varphi_{2, j}\right)\right\|_{\mathcal{B}\left(\left[t_{j-1} ; t_{j}\right] ; Y\right)} \leqslant \alpha_{1}(\delta) \beta_{1}\left(M_{0}\right)\left\|\varphi_{1, j}-\varphi_{2, j}\right\|_{W\left[0 ; t_{j}\right]} .
$$

Therefore,

$$
\left\|\eta_{j}\right\|_{W\left[0 ; t_{j}\right]} \leqslant \mathcal{N}_{\omega} \alpha(\delta) \beta_{1}\left(M_{0}\right)\left\|\eta_{j}\right\|_{W\left[0 ; t_{j}\right]} \leqslant \frac{1}{2}\left\|\eta_{j}\right\|_{W\left[0 ; t_{j}\right]}
$$


Thus,

$$
\frac{1}{2}\left\|\eta_{j}\right\|_{W\left[0 ; t_{j}\right]} \leqslant 0
$$

that is, $\eta_{j}=0$ or $\varphi_{1, j}=\varphi_{2, j}$ in the space $W\left[0 ; t_{j}\right]$.

By induction we conclude that $\varphi_{1}=\varphi_{2}$ in the space $W[0 ; T]$. The proof is complete.

Proof of Theorem 2.2. Let a number $M>0$ be arbitrary and fixed,

$$
\gamma_{0}=\sup _{\tau \in(0 ; T]}\left\|\mathcal{F}_{\tau}(0)\right\|_{W[0 ; \tau]}<\infty
$$

according Condition $\left.\mathbf{G}_{3}\right) ; \aleph_{s}=\max _{t \in[0 ; T]} \alpha_{s}(t), s=0,1$,

$$
\bar{z}=f[\bar{u}](\bar{\varphi}), \quad \gamma_{1}=\|\bar{z}\|_{\mathcal{B}([0 ; T] ; Y)}, \quad M_{0}=\gamma_{0}+\mathcal{N}\left(\gamma_{1}\right) \gamma_{1} \aleph_{0}
$$

We observe that according Conditions $\left.\left.\mathbf{G}_{2}\right), \mathbf{F}_{1}\right)$, for all $\tau \in(0 ; T]$ we have:

$$
\begin{aligned}
\left\|\left.\bar{\varphi}\right|_{[0 ; \tau]}\right\|_{W[0 ; \tau]} & \leqslant\left\|\mathcal{F}_{\tau}(0)\right\|_{W[0 ; \tau]}+\left\|\mathcal{F}_{\tau}\left[\left.\bar{z}\right|_{[0 ; \tau]}\right]-\mathcal{F}_{\tau}(0)\right\|_{W[0 ; \tau]} \\
& \leqslant \gamma_{0}+\mathcal{N}\left(\gamma_{1}\right) \aleph_{0}\|\bar{z}\|_{\mathcal{B}([0 ; \tau] ; Y)} \leqslant M_{0} .
\end{aligned}
$$

We denote

$$
\begin{aligned}
& \aleph_{2}=\aleph_{1} \beta_{1}\left(M_{1}\right)\left\{1+\beta_{0}\left(M_{1}\right)\right\}, \quad \mathcal{N}_{\omega}=\max \{1, \mathcal{N}(\omega)\}, \\
& M_{1}=M+M_{0}, \quad \omega=\gamma_{1}+\left(\beta_{0}\left(M_{1}\right)+1+\aleph_{2}\right) M .
\end{aligned}
$$

We choose a number $\sigma>0$ so that $\sigma \mathcal{N}_{\omega}<1$. After that, employing the continuity of the function $\alpha=\alpha_{0}(\cdot) \alpha_{1}(\cdot)$, see Conditions $\left.\mathbf{G}_{2}\right), \mathbf{F}_{2}$ ), as well as the condition $\alpha(0)=0$, we choose a number $\delta \in\left(0 ; \delta_{0}\right)$ so that

$$
\alpha(\delta) \beta_{1}\left(M_{1}\right)<\sigma .
$$

We choose an arbitrary partition of the segment $[0 ; T]$ of the form

$$
0=t_{0}<t_{1}<\ldots<t_{k}=T, \quad t_{i}-t_{i-1} \leqslant \delta, \quad i=\overline{1, k} .
$$

According Condition $\mathbf{G}_{1}$ ), we denote

$$
\bar{z}_{i}=\left.\bar{z}\right|_{\left[0 ; t_{i}\right]}, \quad \bar{\varphi}_{i}=\left.\bar{\varphi}\right|_{\left[0 ; t_{i}\right]}=\mathcal{F}_{t_{i}}\left[\bar{z}_{i}\right] \in W_{0}\left[0 ; t_{i}\right] .
$$

We note that according Condition $\mathbf{F}_{1}$ ),

$$
\bar{\varphi}_{i}=\mathcal{F}_{t_{i}}\left[f[\bar{u}]\left(\bar{\varphi}_{i}\right)\right], \quad i=\overline{1, k}
$$

We define the function $\ell(c)=\mathcal{N}_{\omega} \aleph_{0}\left[\beta_{0}\left(M_{1}\right)+1+\aleph_{2} c\right]$ and scalar sequences

$$
C_{i}=\left(1+\frac{1}{1-\sigma \mathcal{N}_{\omega}}\right) \ell\left(C_{i-1}\right), \quad \pi_{i}=\frac{\ell\left(C_{i-1}\right)}{1-\sigma \mathcal{N}_{\omega}}, \quad i=\overline{1, k}, \quad C_{0}=0
$$

We choose an arbitrary control $u \in U$ so that

$$
0 \leqslant \rho(u, \bar{u}) \leqslant M, \quad C_{k} \rho(u, \bar{u}) \leqslant M .
$$

Let us show that under such choice, equation $(2.2)$ is solvable.

First of all, we consider the case $\rho(u, \bar{u})=0$. Then $\varphi=\bar{\varphi}$ solves equation (2.2). Indeed, according Condition $\mathbf{G}_{2}$ ),

$$
\begin{aligned}
\|\bar{\varphi}-\mathcal{F}[f[u](\bar{\varphi})]\|_{W[0 ; T]} & =\|\mathcal{F}[f[\bar{u}](\bar{\varphi})]-\mathcal{F}[f[u](\bar{\varphi})]\|_{W[0 ; T]} \\
& \leqslant \mathcal{N}_{\omega} \aleph_{0}\|f[u](\bar{\varphi})-f[\bar{u}](\bar{\varphi})\|_{\mathcal{B}([0 ; T] ; Y)}=\mathcal{N}_{\omega} \aleph_{0} \rho(u, \bar{u})=0 .
\end{aligned}
$$


Here we have taken into account that

$$
\|f[u](\bar{\varphi}) \pm \bar{z}\|_{\mathcal{B}([0 ; T] ; Y)} \leqslant\|\bar{z}\|_{\mathcal{B}([0 ; T] ; Y)}+\rho(u, \bar{u})=\gamma_{1} \leqslant \omega .
$$

This is why in what follows we consider the case $\rho(u, \bar{u})>0$. The further proof is made in several steps. In these steps, we shall consider local analogues of equation 2.2):

$$
\varphi=\mathcal{F}_{t_{j}}[f[u](\varphi)], \quad \varphi \in W\left[0 ; t_{j}\right] .
$$

The solvability of equations $\left(\mathcal{E}_{j}\right)$ in corresponding spaces $W\left[0 ; t_{j}\right]$ will be proved by the induction in $j=\overline{1, k}$.

1. We are going to prove the existence of a function $\varphi=\varphi_{1} \in W_{0}\left[0 ; t_{1}\right]$ solving equation $\left(\mathcal{E}_{1}\right)$ and obeying the estimate:

$$
\left\|\varphi_{1}-\bar{\varphi}_{1}\right\|_{W\left[0 ; t_{1}\right]} \leqslant C_{1} \rho(u, \bar{u}) .
$$

We define $\Psi_{1}$ as a set of all $\psi \in W_{0}\left[0 ; t_{1}\right]$ such that

$$
\left\|\psi-\mathcal{F}_{t_{1}}[\widetilde{z}]\right\|_{W\left[0 ; t_{1}\right]} \leqslant \pi_{1} \rho(u, \bar{u}), \quad \widetilde{z}=f[u]\left(\bar{\varphi}_{1}\right) .
$$

a) Let us show that $\Psi_{1} \neq \emptyset$. Indeed, $\psi=\mathcal{F}_{t_{1}}[\widetilde{z}] \in \Psi_{1}$.

b) Let us show that the set $\Psi_{1}$ is closed in the space $W\left[0 ; t_{1}\right]$. Let $\left\{\eta_{m}\right\} \subset \Psi_{1}$ and

$$
\left\|\eta_{m}-\eta_{0}\right\|_{W\left[0 ; t_{1}\right]} \rightarrow 0 \quad \text { as } \quad m \rightarrow \infty, \quad \eta_{0} \in W\left[0 ; t_{1}\right] .
$$

It is obvious that

$$
\begin{aligned}
\left\|\eta_{0}-\mathcal{F}_{t_{1}}[\widetilde{z}]\right\|_{W\left[0 ; t_{1}\right]} & \leqslant\left\|\eta_{0}-\eta_{m}\right\|_{W\left[0 ; t_{1}\right]}+\left\|\eta_{m}-\mathcal{F}_{t_{1}}[\widetilde{z}]\right\|_{W\left[0 ; t_{1}\right]} \\
& \leqslant\left\|\eta_{m}-\eta_{0}\right\|_{W\left[0 ; t_{1}\right]}+\pi_{1} \rho(u, \bar{u}) .
\end{aligned}
$$

Passing to limit as $m \rightarrow \infty$, we conclude that

$$
\left\|\eta_{0}-\mathcal{F}_{t_{1}}[\widetilde{z}]\right\|_{W\left[0 ; t_{1}\right]} \leqslant \pi_{1} \rho(u, \bar{u}) .
$$

In view of the closedness of the set $W_{0}\left[0 ; t_{1}\right]$ in the space $W\left[0 ; t_{1}\right]$, this means that $\eta_{0} \in \Psi_{1}$. Thus, the set $\Psi_{1}$ is closed in $W\left[0 ; t_{1}\right]$.

c) We define an operator $F_{1}: \Psi_{1} \rightarrow W_{0}\left[0 ; t_{1}\right]$ by the formula

$$
\eta=F_{1}[\varphi]=\mathcal{F}_{t_{1}}[z], \quad \text { where } \quad z=f[u](\varphi), \quad \varphi \in \Psi_{1} .
$$

Let us show that $\eta \in \Psi_{1}$. According Condition $\mathbf{F}_{1}$ ),

$$
\left\|\widetilde{z}-\bar{z}_{1}\right\|_{\mathcal{B}\left(\left[0 ; t_{1}\right] ; Y\right)}=\left\|f[u]\left(\bar{\varphi}_{1}\right)-f[\bar{u}]\left(\bar{\varphi}_{1}\right)\right\|_{\mathcal{B}\left(\left[0 ; t_{1}\right] ; Y\right)} \leqslant \rho(u, \bar{u}) .
$$

Therefore,

$$
\|\widetilde{z}\|_{\mathcal{B}\left(\left[0 ; t_{1}\right] ; Y\right)} \leqslant \gamma_{1}+M \leqslant \omega ; \quad\left\|\bar{z}_{1}\right\|_{\mathcal{B}\left(\left[0 ; t_{1}\right] ; Y\right)} \leqslant \gamma_{1} \leqslant \omega
$$

We estimate:

$$
\left\|\varphi-\bar{\varphi}_{1}\right\|_{W\left[0 ; t_{1}\right]} \leqslant\left\|\varphi-\mathcal{F}_{t_{1}}[\widetilde{z}]\right\|_{W\left[0 ; t_{1}\right]}+\left\|\mathcal{F}_{t_{1}}[\widetilde{z}]-\mathcal{F}_{t_{1}}\left[\bar{z}_{1}\right]\right\|_{W\left[0 ; t_{1}\right]} .
$$

Hence, employing the definition of the set $\Psi_{1}$ and Condition $\mathbf{G}_{2}$ ), we obtain:

$$
\left\|\varphi-\bar{\varphi}_{1}\right\|_{W\left[0 ; t_{1}\right]} \leqslant \pi_{1} \rho(u, \bar{u})+\mathcal{N}_{\omega} \aleph_{0}\left\|\widetilde{z}-\bar{z}_{1}\right\|_{\mathcal{B}\left(\left[0 ; t_{1}\right] ; Y\right)} \leqslant\left[\pi_{1}+\ell(0)\right] \rho(u, \bar{u}),
$$

that is,

$$
\left\|\varphi-\bar{\varphi}_{1}\right\|_{W\left[0 ; t_{1}\right]} \leqslant C_{1} \rho(u, \bar{u}) \leqslant M .
$$

Therefore,

$$
\|\varphi\|_{W\left[0 ; t_{1}\right]} \leqslant\left\|\bar{\varphi}_{1}\right\|_{W\left[0 ; t_{1}\right]}+M \leqslant M_{0}+M=M_{1} .
$$


According Condition $\mathbf{F}_{2}$ ),

$$
\begin{aligned}
\|z\|_{\mathcal{B}\left(\left[0 ; t_{1}\right] ; Y\right)} & =\left\|f[u](\varphi) \pm f[u]\left(\bar{\varphi}_{1}\right)\right\|_{\mathcal{B}\left(\left[0 ; t_{1}\right] ; Y\right)} \\
& \leqslant \aleph_{1} \beta_{1}\left(M_{1}\right)\left\|\varphi-\bar{\varphi}_{1}\right\|_{W\left[0 ; t_{1}\right]}+\|\widetilde{z}\|_{\mathcal{B}\left(\left[0 ; t_{1}\right] ; Y\right)} \\
& \leqslant \aleph_{1} \beta_{1}\left(M_{1}\right) M+M+\gamma_{1} \leqslant \omega .
\end{aligned}
$$

Employing Conditions $\mathbf{G}_{2}$ ), $\mathbf{F}_{2}$ ), we obtain:

$$
\begin{aligned}
\left\|\eta-\mathcal{F}_{t_{1}}[\widetilde{z}]\right\|_{W\left[0 ; t_{1}\right]} & =\left\|\mathcal{F}_{t_{1}}[z]-\mathcal{F}_{t_{1}}[\widetilde{z}]\right\|_{W\left[0 ; t_{1}\right]} \\
& \leqslant \mathcal{N}_{\omega} \alpha_{0}(\delta)\left\|f[u](\varphi)-f[u]\left(\bar{\varphi}_{1}\right)\right\|_{\mathcal{B}\left(\left[0 ; t_{1}\right] ; Y\right)} \\
& \leqslant \mathcal{N}_{\omega} \alpha(\delta) \beta_{1}\left(M_{1}\right)\left\|\varphi-\bar{\varphi}_{1}\right\|_{W\left[0 ; t_{1}\right]} .
\end{aligned}
$$

Since $\sigma \mathcal{N}_{\omega}<1$, this yields that

$$
\left\|\eta-\mathcal{F}_{t_{1}}[\widetilde{z}]\right\|_{W\left[0 ; t_{1}\right]} \leqslant\left[\sigma \mathcal{N}_{\omega} \pi_{1}+\ell\left(C_{0}\right)\right] \rho(u, \bar{u})=\pi_{1} \rho(u, \bar{u}) .
$$

Therefore, $\eta \in \Psi_{1}$ and thus, $F_{1}: \Psi_{1} \rightarrow \Psi_{1}$.

d) Let us establish that the operator $F_{1}$ is contracting on the set $\Psi_{1}$. We choose arbitrary $\psi_{1}, \psi_{2} \in \Psi_{1}$ and we let

$$
z_{s}=f[u]\left(\psi_{s}\right)(t), \quad t \in\left[0 ; t_{1}\right], \quad \eta_{s}=F_{1}\left[\psi_{s}\right]=\mathcal{F}_{t_{1}}\left[z_{s}\right] \in \Psi_{1}, \quad s=1,2 .
$$

Similar to Subsection 1,c), we have:

$$
\left\|z_{s}\right\|_{\mathcal{B}\left(\left[0 ; t_{1}\right] ; Y\right)} \leqslant \omega, \quad\left\|\eta_{s}\right\|_{W\left[0 ; t_{1}\right]} \leqslant M_{1}, \quad s=1,2 .
$$

Employing Condition $\mathbf{G}_{2}$ ), we estimate as follows:

$$
\left\|\eta_{1}-\eta_{2}\right\|_{W\left[0 ; t_{1}\right]} \leqslant \mathcal{N}_{\omega} \alpha_{0}(\delta)\left\|z_{1}-z_{2}\right\|_{\mathcal{B}\left(\left[0 ; t_{1}\right] ; Y\right)},
$$

where, according Condition $\mathbf{F}_{2}$ ),

$$
\left\|z_{1}-z_{2}\right\|_{\mathcal{B}\left(\left[0 ; t_{1}\right] ; Y\right)} \leqslant \alpha_{1}(\delta) \beta_{1}\left(M_{1}\right)\left\|\psi_{1}-\psi_{2}\right\|_{W\left[0 ; t_{1}\right]} .
$$

Therefore,

$$
\left\|\eta_{1}-\eta_{2}\right\|_{W\left[0 ; t_{1}\right]} \leqslant \mathcal{N}_{\omega} \alpha(\delta) \beta_{1}\left(M_{1}\right)\left\|\psi_{1}-\psi_{2}\right\|_{W\left[0 ; t_{1}\right]} \leqslant \mathcal{N}_{\omega} \sigma\left\|\psi_{1}-\psi_{2}\right\|_{W\left[0 ; t_{1}\right]},
$$

where $\mathcal{N}_{\omega} \sigma<1$. This means that the operator $F_{1}$ is contracting on the set $\Psi_{1}$.

e) According the contracting mapping principle, we conclude that equation $\left(\mathcal{E}_{1}\right)$ is uniquely solvable in the set $\Psi_{1}$, that is, it possesses a solution $\varphi_{1} \in W_{0}\left[0 ; t_{1}\right]$ satisfying the estimate:

$$
\left\|\varphi_{1}-\mathcal{F}_{t_{1}}[\widetilde{z}]\right\|_{W\left[0 ; t_{1}\right]} \leqslant \pi_{1} \rho(u, \bar{u})
$$

As it was shown in Subsection 1,c), the belonging $\varphi_{1} \in \Psi_{1}$ implies the inequalities:

$$
\left\|\varphi_{1}-\bar{\varphi}_{1}\right\|_{W\left[0 ; t_{1}\right]} \leqslant C_{1} \rho(u, \bar{u}), \quad\left\|\varphi_{1}\right\|_{W\left[0 ; t_{1}\right]} \leqslant M_{1} .
$$

2. Proceeding by induction, we assume that we have already proved the existence of a solution $\varphi_{j-1} \in W_{0}\left[0 ; t_{j-1}\right]$ to equation $\left(\mathcal{E}_{j-1}\right), j \in \overline{2, m}$, obeying the estimates:

$$
\left\|\varphi_{j-1}\right\|_{W\left[0 ; t_{j-1}\right]} \leqslant M_{1},\left\|\varphi_{j-1}-\bar{\varphi}_{j-1}\right\|_{W\left[0 ; t_{j-1}\right]} \leqslant C_{j-1} \rho(u, \bar{u}) .
$$

We are going to prove that this assumption implies a similar statement for equation $\left(\mathcal{E}_{j}\right)$.

We define $\Psi_{j}$ as the set of all $\psi \in \Phi\left[t_{j}, \varphi_{j-1}\right]$ such that

$$
\left\|\psi-\mathcal{F}_{t_{j}}[\widetilde{z}]\right\|_{W\left[0 ; t_{j}\right]} \leqslant \pi_{j} \rho(u, \bar{u}), \quad \widetilde{z}=Z\left[t_{j-1}, t_{j}, \gamma, u, \bar{u}, \varphi_{j-1}, \bar{\varphi}_{j}\right],
$$

and in view of Condition $\mathbf{F}_{3}$ ),

$$
\widetilde{z} \in \mathcal{B}\left(\left[0 ; t_{j}\right] ; Y\right),\left.\quad \widetilde{z}\right|_{\left[0 ; t_{j-1}\right]}=f[u]\left(\varphi_{j-1}\right), \quad \gamma=\rho(u, \bar{u}) .
$$


a) Let us show that $\Psi_{j} \neq \emptyset$. Indeed, $\psi=\mathcal{F}_{t_{j}}[\widetilde{z}] \in W_{0}\left[0 ; t_{j}\right]$ obeys estimate $(3.3)$ and by Condition $\mathbf{G}_{1}$ ),

$$
\left.\psi\right|_{\left[0 ; t_{j-1}\right]}=\mathcal{F}_{t_{j-1}}\left[\left.\widetilde{z}\right|_{\left[0 ; t_{j-1}\right]}\right]=\mathcal{F}_{t_{j-1}}\left[f[u]\left(\varphi_{j-1}\right)\right]=\varphi_{j-1} .
$$

Therefore, $\psi \in \Phi\left[t_{j}, \varphi_{j-1}\right]$, and hence, $\psi \in \Psi_{j}$.

b) In view of Lemma 3.1, the closedness of the set $\Psi_{j}$ can be proven in the same way how this was done for the set $\Psi_{1}$.

c) We define an operator $F_{j}: \Psi_{j} \rightarrow W_{0}\left[0 ; t_{j}\right]$ by the formula

$$
\eta=F_{j}[\varphi]=\mathcal{F}_{t_{j}}[z], \quad \text { where } \quad z=f[u](\varphi), \quad \varphi \in \Psi_{j} .
$$

Let us show that $\eta \in \Psi_{j}$. According (3.3) and Conditions $\left.\left.\mathbf{F}_{3}\right), \mathbf{F}_{1}\right), \mathbf{F}_{2}$ ),

$$
\begin{aligned}
\left\|\widetilde{z}-\bar{z}_{j}\right\|_{\mathcal{B}\left(\left[t_{j-1} ; t_{j}\right] ; Y\right)} & \leqslant \beta_{0}\left(M_{1}\right)\left(\rho(u, \bar{u})+\left\|f[u]\left(\varphi_{j-1}\right)-f[u]\left(\bar{\varphi}_{j-1}\right)\right\|_{\mathcal{B}\left(\left[0 ; t_{j-1}\right] ; Y\right)}\right) \\
& \leqslant \beta_{0}\left(M_{1}\right)\left\{\rho(u, \bar{u})+\beta_{1}\left(M_{1}\right) \aleph_{1}\left\|\varphi_{j-1}-\bar{\varphi}_{j-1}\right\|_{W\left[0 ; t_{j-1}\right]}\right\} .
\end{aligned}
$$

In the same, according Conditions $\left.\mathbf{F}_{2}\right), \mathbf{F}_{1}$ ),

$$
\begin{aligned}
\left\|\widetilde{z}-\bar{z}_{j}\right\|_{\mathcal{B}\left(\left[0 ; t_{j-1}\right] ; Y\right)} & =\left\|f[u]\left(\varphi_{j-1}\right)-f[\bar{u}]\left(\bar{\varphi}_{j-1}\right) \pm f[u]\left(\bar{\varphi}_{j-1}\right)\right\|_{\mathcal{B}\left(\left[0 ; t_{j-1}\right] ; Y\right)} \\
& \leqslant \beta_{1}\left(M_{1}\right) \aleph_{1}\left\|\varphi_{j-1}-\bar{\varphi}_{j-1}\right\|_{W\left[0 ; t_{j-1}\right]}+\rho(u, \bar{u}) .
\end{aligned}
$$

Thus,

$$
\left\|\widetilde{z}-\bar{z}_{j}\right\|_{\mathcal{B}\left(\left[0 ; t_{j}\right] ; Y\right)} \leqslant\left\{\beta_{0}\left(M_{1}\right)+1\right\} \rho(u, \bar{u})+\aleph_{2}\left\|\varphi_{j-1}-\bar{\varphi}_{j-1}\right\|_{W\left[0 ; t_{j-1}\right]}
$$

and by the induction assumption,

$$
\left\|\widetilde{z}-\bar{z}_{j}\right\|_{\mathcal{B}\left(\left[0 ; t_{j}\right] ; Y\right)} \leqslant\left\{\beta_{0}\left(M_{1}\right)+1+\aleph_{2} C_{j-1}\right\} \rho(u, \bar{u})=\frac{\ell\left(C_{j-1}\right)}{\mathcal{N}_{\omega} \aleph_{0}} \rho(u, \bar{u}) .
$$

In particular, taking into consideration that

$$
C_{j-1} \rho(u, \bar{u}) \leqslant C_{k} \rho(u, \bar{u}) \leqslant M, \quad \rho(u, \bar{u}) \leqslant M,
$$

we have

$$
\left\|\widetilde{z}-\bar{z}_{j}\right\|_{\mathcal{B}\left(\left[0 ; t_{j}\right] ; Y\right)} \leqslant\left[\beta_{0}\left(M_{1}\right)+1+\aleph_{2}\right] M
$$

Therefore,

$$
\|\widetilde{z}\|_{\mathcal{B}\left(\left[0 ; t_{j}\right] ; Y\right)} \leqslant \gamma_{1}+\left[\beta_{0}\left(M_{1}\right)+1+\aleph_{2}\right] M \leqslant \omega, \quad\left\|\bar{z}_{j}\right\|_{\mathcal{B}\left(\left[0 ; t_{j}\right] ; Y\right)} \leqslant \gamma_{1} \leqslant \omega .
$$

We estimate:

$$
\left\|\varphi-\bar{\varphi}_{j}\right\|_{W\left[0 ; t_{j}\right]} \leqslant\left\|\varphi-\mathcal{F}_{t_{j}}[\widetilde{z}]\right\|_{W\left[0 ; t_{j}\right]}+\left\|\mathcal{F}_{t_{j}}[\widetilde{z}]-\mathcal{F}_{t_{j}}\left[\bar{z}_{j}\right]\right\|_{W\left[0 ; t_{j}\right]} .
$$

By the definition of the set $\Psi_{j}$ and Condition $\mathbf{G}_{2}$ ), we obtain:

$$
\left\|\varphi-\bar{\varphi}_{j}\right\|_{W\left[0 ; t_{j}\right]} \leqslant \pi_{j} \rho(u, \bar{u})+\mathcal{N}_{\omega} \aleph_{0}\left\|\widetilde{z}-\bar{z}_{j}\right\|_{\mathcal{B}\left(\left[0 ; t_{j}\right] ; Y\right)} \leqslant\left[\pi_{j}+\ell\left(C_{j-1}\right)\right] \rho(u, \bar{u}),
$$

that is,

Hence,

$$
\left\|\varphi-\bar{\varphi}_{j}\right\|_{W\left[0 ; t_{j}\right]} \leqslant C_{j} \rho(u, \bar{u}) \leqslant M .
$$

According Conditions $\mathbf{F}_{2}$ ), $\mathbf{F}_{1}$ ),

$$
\begin{aligned}
\|z\|_{\mathcal{B}\left(\left[0 ; t_{j}\right] ; Y\right)} & =\left\|f[u](\varphi) \pm f[u]\left(\bar{\varphi}_{j}\right)\right\|_{\mathcal{B}\left(\left[0 ; t_{j}\right] ; Y\right)} \\
& \leqslant \aleph_{1} \beta_{1}\left(M_{1}\right)\left\|\varphi-\bar{\varphi}_{j}\right\|_{W\left[0 ; t_{j}\right]}+\left\|f[u]\left(\bar{\varphi}_{j}\right) \pm f[\bar{u}]\left(\bar{\varphi}_{j}\right)\right\|_{\mathcal{B}\left(\left[0 ; t_{j}\right] ; Y\right)} \\
& \leqslant \aleph_{1} \beta_{1}\left(M_{1}\right) M+M+\gamma_{1} \leqslant \omega .
\end{aligned}
$$


Since

$$
\varphi \in \Phi\left[t_{j}, \varphi_{j-1}\right], \quad \psi=\mathcal{F}_{t_{j}}[\widetilde{z}] \in \Phi\left[t_{j}, \varphi_{j-1}\right]
$$

see Subsection 2,a), by Lemma 3.2, see (3.1), we get:

$$
\left.z\right|_{\left[0 ; t_{j-1}\right]}=\left.f[u](\varphi)\right|_{\left[0 ; t_{j-1}\right]}=\left.f[u](\psi)\right|_{\left[0 ; t_{j-1}\right]} .
$$

At that, as it was shown in Subsection 2,a), $\left.\psi\right|_{\left[0 ; t_{j-1}\right]}=\varphi_{j-1}$. Hence, according Condition $\mathbf{F}_{1}$ ),

$$
\left.z\right|_{\left[0 ; t_{j-1}\right]}=f[u]\left(\varphi_{j-1}\right)=\left.\widetilde{z}\right|_{\left[0 ; t_{j-1}\right]} .
$$

In view of the established facts, we employ Conditions $\mathbf{G}_{2}$ ), $\mathbf{F}_{2}$ ), we get:

$$
\begin{aligned}
\left\|\eta-\mathcal{F}_{t_{j}}[\widetilde{z}]\right\|_{W\left[0 ; t_{j}\right]} & =\| \mathcal{F}_{t_{j}}[z]-\mathcal{F}_{t_{j}}\left[\widetilde{z} \|_{W\left[0 ; t_{j}\right]}\right. \\
& \leqslant \mathcal{N}_{\omega} \alpha_{0}(\delta)\left\|f[u](\varphi)-f[u]\left(\bar{\varphi}_{j}\right)\right\|_{\mathcal{B}\left(\left[t_{j-1} ; t_{j}\right] ; Y\right)} \\
& \leqslant \mathcal{N}_{\omega} \alpha(\delta) \beta_{1}\left(M_{1}\right)\left\|\varphi-\bar{\varphi}_{j}\right\|_{W\left[0 ; t_{j}\right]} .
\end{aligned}
$$

Hence, by 3.4 and the inequalities $\alpha(\delta) \beta_{1}\left(M_{1}\right) \leqslant \sigma, \sigma \mathcal{N}_{\omega}<1$, we get:

$$
\left\|\eta-\mathcal{F}_{t_{j}}[\widetilde{z}]\right\|_{W\left[0 ; t_{j}\right]} \leqslant\left[\sigma \mathcal{N}_{\omega} \pi_{j}+\ell\left(C_{j-1}\right)\right] \rho(u, \bar{u})=\pi_{j} \rho(u, \bar{u}) .
$$

Thus, $\eta$ obeys estimate $(3.3)$.

Let us show that $\eta \in \Phi\left[t_{j}, \varphi_{j-1}\right]$. Indeed, by $(3.5)$, Condition $\mathbf{G}_{1}$ ) and induction assumption, we conclude that this property holds in a stronger form:

$$
\left.\eta\right|_{\left[0 ; t_{j-1}\right]}=\mathcal{F}_{t_{j-1}}\left[\left.z\right|_{\left[0 ; t_{j-1}\right]}\right]=\mathcal{F}_{t_{j-1}}\left[f[u]\left(\varphi_{j-1}\right)\right]=\varphi_{j-1}
$$

Thus, $\eta \in \Psi_{j}$, and hence, $F_{j}: \Psi_{j} \rightarrow \Psi_{j}$.

d) Let us show that the operator $F_{j}$ is contracting on the set $\Psi_{j}$. We choose arbitrary $\psi_{1}$, $\psi_{2} \in \Psi_{j}$ and we let

$$
z_{s}=f[u]\left(\psi_{s}\right), \quad \eta_{s}=F_{j}\left[\psi_{s}\right]=\mathcal{F}_{t_{j}}\left[z_{s}\right], \quad s=1,2 .
$$

Similar to Subsection 2,c) we have:

$$
\left\|z_{s}\right\|_{\mathcal{B}\left(\left[0 ; t_{j}\right] ; Y\right)} \leqslant \omega, \quad\left\|\eta_{s}\right\|_{W\left[0 ; t_{j}\right]} \leqslant M_{1}, \quad s=1,2 .
$$

In view of Lemma 3.2 , see 3.1 , we get:

$$
\left.z_{1}\right|_{\left[0 ; t_{j-1}\right]}=\left.f[u]\left(\psi_{1}\right)\right|_{\left[0 ; t_{j-1}\right]}=\left.f[u]\left(\psi_{2}\right)\right|_{\left[0 ; t_{j-1}\right]}=\left.z_{2}\right|_{\left[0 ; t_{j-1}\right]}
$$

since $\psi_{s} \in \Phi\left[t_{j}, \varphi_{j-1}\right], s=1,2$. This is why, by employing Conditions $\left.\mathbf{G}_{2}\right), \mathbf{F}_{2}$ ), we obtain:

$$
\begin{aligned}
\left\|\eta_{1}-\eta_{2}\right\|_{W\left[0 ; t_{j}\right]} & \leqslant \mathcal{N}_{\omega} \alpha_{0}(\delta)\left\|z_{1}-z_{2}\right\|_{\mathcal{B}\left(\left[t_{j-1} ; t_{j}\right] ; Y\right)}=\mathcal{N}_{\omega} \alpha_{0}(\delta)\left\|f[u]\left(\psi_{1}\right)-f[u]\left(\psi_{2}\right)\right\|_{\mathcal{B}\left(\left[t_{j-1} ; t_{j}\right] ; Y\right)} \\
& \leqslant \mathcal{N}_{\omega} \alpha(\delta) \beta_{1}\left(M_{1}\right)\left\|\psi_{1}-\psi_{2}\right\|_{W\left[0 ; t_{j}\right]} \leqslant \mathcal{N}_{\omega} \sigma\left\|\psi_{1}-\psi_{2}\right\|_{W\left[0 ; t_{j}\right]},
\end{aligned}
$$

where $\mathcal{N}_{\omega} \sigma<1$. This means that the operator $F_{j}$ is contracting on the set $\Psi_{j}$.

e) By the contracting mapping principle, we conclude that equation $\left(\mathcal{E}_{j}\right)$ has a unique solution in the set $\Psi_{j}$, that is, a solution $\varphi_{j} \in \Phi\left[t_{j}, \varphi_{j-1}\right]$ satisfying the estimates

$$
\left\|\varphi_{j}-\bar{\varphi}_{j}\right\|_{W\left[0 ; t_{j}\right]} \leqslant C_{j} \rho(u, \bar{u}), \quad\left\|\varphi_{j}\right\|_{W\left[0 ; t_{j}\right]} \leqslant M_{1},
$$

which are obtained are similar to $(3.4)$ just by using that $\varphi_{j} \in \Psi_{j}$. Moreover, similar to relation (3.6) we obtain: $\left.\varphi_{j}\right|_{\left[0 ; t_{j-1}\right]}=\varphi_{j-1}$.

3. By induction we conclude that a similar statement holds for $j=k$. And this means that equation 2.2 has a solution $\varphi \in W_{0}[0 ; T]$ satisfying the estimates:

$$
\|\varphi\|_{W[0 ; T]} \leqslant M_{1}, \quad\|\varphi-\bar{\varphi}\|_{W[0 ; T]} \leqslant C_{k} \rho(u, \bar{u}) .
$$


The uniqueness of solution in the space $W[0 ; T]$ follows Theorem 2.1. The proof is complete.

\section{EXAMPLE: initial BOUNDARY VALUE PROBLEM FOR NAVIER-STOKES SYSTEM}

Let $\Omega \subset \mathbb{R}^{3}$ be a bounded domain with a boundary $\partial \Omega=S \in C^{2}, T>0, p, r \geqslant 1, Q_{T}=$ $\Omega \times[0 ; T]$. Following [31], apart of standard Lebesgue spaces $L_{p}(\Omega), L_{p}\left(Q_{T}\right)$ and anisotropic Lebesgue spaces $L_{p, r}\left(Q_{T}\right)=L_{r}\left([0 ; T] ; L_{p}(\Omega)\right)$, we shall make use of the following functional spaces.

1) The Sobolev space $W[0 ; T]=W_{p}^{2,1}\left(Q_{T}\right)$ with the norm

$$
\|\varphi\|_{W[0 ; T]}=\sum_{|\mu| \leqslant 2}\left\|\partial^{\mu} \varphi\right\|_{p, Q_{T}}+\left\|\partial_{t} \varphi\right\|_{p, Q_{T}}
$$

where $\|\cdot\|_{p, Q_{T}}=\|\cdot\|_{L_{p}\left(Q_{T}\right)}, \mu=\left(\mu_{1}, \mu_{2}, \mu_{3}\right)$ is a multi-index,

$$
|\mu|=\sum_{i=1}^{3} \mu_{i}, \quad \partial^{\mu} \varphi=\frac{\partial^{|\mu|} \varphi}{\partial x_{1}^{\mu_{1}} \partial x_{2}^{\mu_{2}} \partial x_{3}^{\mu_{3}}}, \quad \partial_{t} \varphi=\frac{\partial \varphi}{\partial t},
$$

where all derivatives are treated in the generalized sense. We shall also employ the following notation for the norm: $\|\cdot\|_{p, r, Q_{T}}=\|\cdot\|_{L_{p, r}\left(Q_{T}\right)}$.

2) The symbol $\stackrel{\circ}{j}_{p}^{2-\frac{2}{p}}(\Omega)$ denotes the closure of the set of smooth compactly supported solenoidal vectors vanishing on $S$ with respect to the norm $W_{p}^{2-\frac{2}{p}}(\Omega)$, see. $\mid 31$. An equivalent norm in $\stackrel{\circ}{p}^{2-\frac{2}{p}}(\Omega)$ is

$$
\|\varphi\|_{p, \Omega}=\inf _{\psi}\|\psi\|_{W[0 ; 1]}
$$

where the infimum is taken over all continuations $\psi(x, t)$ of a vector $\varphi$ on the set $Q_{1}$. It can be shown that for each given $\varphi$ in the considered space at least one mentioned continuation exists.

3) The classes $G_{p}(\Omega)$ and $\stackrel{\circ}{J}_{p}(\Omega)$ are defined as follows $\mid 31$. Each smooth compactly supported vector $f(x)$ can be represented as a sum of two terms orthogonal in $L_{2}(\Omega)$ :

$$
f(x)=\nabla \varphi(x)+g(x), \quad \text { where } \quad \nabla \cdot g=0,\left.\quad g \cdot n(x)\right|_{S}=0,
$$

$n(x)$ is the unit outward normal,

$$
\Delta \varphi=\nabla \cdot f(x),\left.\quad \frac{\partial \varphi}{\partial n}\right|_{S}=\left.f \cdot n\right|_{S}
$$

and $\nabla \varphi$ and $g$ for all $\nabla \Phi \in L_{p^{\prime}}(\Omega)$ satisfy the identities:

$$
(\nabla \varphi, \nabla \Phi)=(f, \nabla \Phi), \quad(g, \nabla \Phi)=0
$$

where

$$
(\varphi, \psi)=\int_{\Omega} \varphi \cdot \psi d x=\sum_{i=1}^{3} \int_{\Omega} \varphi_{i}(x) \psi_{i}(x) d x .
$$

By $G_{p}(\Omega)$ we denote the set of all $\nabla \varphi \in L_{p}(\Omega)$, while $\stackrel{\circ}{J}_{p}(\Omega)$ stands for the set of all vectors in $g \in L_{p}(\Omega)$ obeying identity 4.2$)$. We note that in $|31|$ the notations $L_{p}^{3}(\Omega)$ for the space of vector functions and $L_{p}(\Omega)$ for the space of scalar functions are not distinguished. This is why, to have a better accordance with [31], we also adopt the same convention.

4) The classes $G_{p}\left(Q_{T}\right)$ and $\dot{J}_{p}\left(Q_{T}\right)$ are subspaces of $L_{p}\left(Q_{T}\right)$ consisting of the vectors belonging to $G_{p}(\Omega)$ and $\stackrel{\circ}{J}_{p}(\Omega)$ for almost each $t \in[0 ; T]$. Relation $(4.1)$ means that

$$
L_{p}(\Omega)=G_{p}(\Omega) \oplus \stackrel{\circ}{\dot{J}_{p}}(\Omega), \quad L_{p}\left(Q_{T}\right)=G_{p}\left(Q_{T}\right) \oplus \stackrel{\circ}{J}_{p}\left(Q_{T}\right) .
$$

Following [31], we shall employ the notations: $P_{G}$ is the operator of projecting $L_{p}\left(Q_{T}\right) \rightarrow$ $G_{p}\left(Q_{T}\right), P_{J}$ is the operator of projecting $L_{p}\left(Q_{T}\right) \rightarrow \stackrel{\circ}{J}_{p}\left(Q_{T}\right)$. 
First of all, following [31], we consider an initial boundary value problem for a linearized Navier-Stokes system:

$$
\begin{aligned}
& \frac{\partial \varphi}{\partial t}-\nu \Delta \varphi+a(x, t) \varphi+\sum_{k=1}^{3} a_{k}(x, t) \varphi_{x_{k}}+\nabla \mathcal{P}=z(x, t), \quad x \in \Omega, \quad t \in[0 ; T], \\
& \nabla \cdot \varphi=0, \quad \text { that is } \operatorname{div} \varphi=0, \\
& \left.\varphi\right|_{x \in S}=0, \quad t \in[0 ; T] ;\left.\quad \varphi\right|_{t=0}=\varphi_{0}(x), \quad x \in \Omega .
\end{aligned}
$$

Here $\varphi(x, t)=\left(\varphi_{1}(x, t), \varphi_{2}(x, t), \varphi_{3}(x, t)\right)$ is the velocity field of a liquid, $\mathcal{P}(x, t)$ is a pressure, $\varphi$ and $\mathcal{P}$ are unknowns, $a(x, t), a_{k}(x, t)$ are given functions such that

$$
\|a\|_{s, \sigma, Q_{T}}+\sum_{j=1}^{3}\left\|a_{j}\right\|_{s_{1}, \sigma_{1}, Q_{T}} \leqslant M
$$

where

$$
\|a\|_{s, \sigma, Q_{T}}=\max _{k, m}\left\|a^{k, m}\right\|_{s, \sigma, Q_{T}}, \quad \frac{3}{2 s}+\frac{1}{\sigma}<1, \quad \frac{3}{2 s_{1}}+\frac{1}{\sigma_{1}}<\frac{1}{2} ;
$$

$z=\left(z_{1}, z_{2}, z_{3}\right) \in L_{p}\left(Q_{T}\right)$ is the density of external fields, $\nu$ is the viscosity coefficient. In [31] a solution to problem (4.3)-(4.5) was constructed in two ways:

1) as a limit of classical solutions in the norm of an appropriate space [31, Sect. 4];

2 ) as a solution to Cauchy problem for an evolution operator differential equation in the space $\stackrel{\circ}{J}_{p}(\Omega)$.

But the result, namely, the conditions of the existence and the uniqueness and the estimates for the solutions, is the same for both ways.

Given a function $z \in L_{p}\left(Q_{T}\right)$, we get: $z=P_{G} z+P_{J} z$. Here $P_{G} z$ is added to the vector $\nabla \mathcal{P}$, and after that $z$ in the right hand side is treated as $P_{J} z$. We note that the operator $P_{J}$ is bounded, see [31, Thm. 2.2].

A solution to problem (4.3)-4.5) is treated as in [31, Sect. 5]. According that approach, we seek it as a pair $\varphi \in W[0 ; T], \nabla \mathcal{P} \in L_{p}\left(Q_{T}\right)$. The following statement holds $[31$, Thm. 4.2, Cor. 2, Sect. 5].

Lemma 4.1. Under the above assumptions, for each pair $z \in L_{p}\left(Q_{T}\right), \varphi_{0} \in{\stackrel{\circ}{J_{p}}}^{2-\frac{2}{p}}(\Omega)$ problem (4.3) - (4.5) possesses a unique solution $\varphi \in W[0 ; T], \nabla \mathcal{P} \in L_{p}\left(Q_{T}\right)$, and the estimate holds true:

$$
\|\varphi\|_{W[0 ; T]}+\|\nabla \mathcal{P}\|_{p, Q_{T}} \leqslant C_{1}\left(1+e^{\gamma T}\right)\left\{\left\|\varphi_{0}\right\|_{p, \Omega}+\|z\|_{p, Q_{T}}\right\}
$$

where $C_{1}, \gamma>0$ are constants independent of $T, \varphi_{0}, z$.

Remark 4.1. The proof of estimate (4.6] in [31] employs implicitly, with no explanations, an inequality of the form

$$
q b^{q-1}\left(c_{1} a+c_{2} b\right) \leqslant \gamma q b^{q}+c_{3} a^{q} \quad \text { for all } \quad a, b>0
$$

as $\gamma>c_{2}$. In the next statement we establish this inequality.

Lemma 4.2. Let $\gamma>c_{2}, c_{1}, c_{2}>0, q \geqslant 1$. There exists a constant $c_{3}=c_{3}\left(q, \gamma, c_{1}, c_{2}\right)>0$ such that inequality (4.7) holds for all $a, b \geqslant 0$.

Proof. In the cases $a=0, b=0, q=1$ the statement is trivial. This is why in suppose that $a>0, b>0, q>1$. We consider the left hand side of inequality (4.7):

$$
q b^{q-1}\left(c_{1} a+c_{2} b\right)=\gamma q b^{q}+\left(\gamma-c_{2}\right) q b^{q-1}\left(\frac{c_{1}}{\gamma-c_{2}} a-b\right) .
$$


We denote $\beta=\frac{c_{1}}{\gamma-c_{2}}$. It is sufficient to show that

$$
\beta a b^{q-1}-b^{q} \leqslant \alpha a^{q}
$$

that is,

$$
\beta\left(\frac{a}{b}\right) \leqslant \alpha\left(\frac{a}{b}\right)^{q}+1 \quad \text { for all } a, b>0,
$$

for some $\alpha>0$. In this case, inequality (4.7) holds true with $c_{3}=\alpha q\left(\gamma-c_{2}\right)$.

We consider the function $f(t)=1+t^{q}-\beta t$. It is clear that $f(0)=1>0$, and by the theorem on stability of the sign of a continuous function, there exists $\bar{t}>0$ such that $f(t)>0$ for all $t \in[0 ; \bar{t}]$.

In what follows we consider two cases:

1) $t=\frac{a}{b}<\bar{t}$. Then (4.8) holds as $\alpha=1$, and thus, 4.7) holds true.

2) $t=\frac{a}{b} \geqslant \bar{t}$, that is, $b \leqslant \frac{a}{\bar{t}}$. Therefore,

$$
q b^{q-1}\left(c_{1} a-\left(\gamma-c_{2}\right) b\right) \leqslant q b^{q-1} c_{1} a \leqslant \frac{q}{\bar{t}^{q-1}} c_{1} a^{q},
$$

that is, 4.7 holds for $c_{3}=\frac{q c_{1}}{\bar{t}^{q-1}}$.

Thus, we can take $c_{3}=\max \left\{q\left(\gamma-c_{2}\right), \frac{q c_{1}}{\bar{t}^{q-1}}\right\}$. The proof is complete.

The statement and the proof of Theorem 2.1 in [31] and the proof of Lemma 10.1 in [31] imply the following lemma.

Lemma 4.3. Let $\tau \in(0 ; \tau], \varphi \in W[0 ; \tau]$. If $q_{1}, r_{1}, q_{2}, r_{2} \geqslant p>1$ are such that the conditions

$$
\rho_{1}=1-\frac{5}{2 p}+\frac{3}{2 q_{1}}+\frac{1}{r_{1}} \geqslant 0, \quad \rho_{2}=\frac{1}{2}-\frac{5}{2 p}+\frac{3}{2 q_{2}}+\frac{1}{r_{2}} \geqslant 0,
$$

hold, where as $p_{i}=\infty$ or $r_{i}=\infty, i=1,2$, the inequality are assumed to be strict, then the estimates

$$
\begin{aligned}
& \left\|\varphi_{i}\right\|_{q_{1}, r_{1}, Q_{\tau}} \leqslant c\left\{\|\varphi\|_{W[0 ; \tau]}+\|\varphi(\cdot, 0)\|_{p, \Omega}\right\}, \\
& \sum_{i=1}^{3}\left\|\varphi_{x_{i}}\right\|_{q_{2}, r_{2}, Q_{\tau}} \leqslant c\left\{\|\varphi\|_{W[0 ; \tau]}+\|\varphi(\cdot, 0)\|_{p, \Omega}\right\},
\end{aligned}
$$

hold true. Here $c$ is a constant independent of $\tau, \varphi$ but depending of the domain $\Omega$.

Let $\tau \in(0 ; T], \varphi_{0} \in{\stackrel{\circ}{J_{p}^{2}}}^{-\frac{2}{p}}(\Omega)$ be fixed but arbitrary. We denote $Y=L_{p}(\Omega), W_{0}[0 ; \tau]$ is the set of all $\varphi \in W[0 ; \tau]$ such that

$$
\left\|\varphi(\cdot, 0)-\varphi_{0}\right\|_{p, \Omega}=0
$$

It is clear that

$$
L_{p}\left(Q_{\tau}\right)=L_{p}([0 ; \tau] ; Y) \equiv \mathcal{B}([0 ; \tau] ; Y) .
$$

The numbers $q_{1}, r_{1}, q_{2}, r_{2}$ are chosen as in Lemma 4.3. We define an operator $\mathcal{F}_{\tau}: L_{p}\left(Q_{\tau}\right) \rightarrow$ $W_{0}[0 ; \tau]$ as follows. Given $z \in L_{p}\left(Q_{\tau}\right)$, we find a pair $\varphi \in W_{0}[0 ; \tau]$ and $\nabla \mathcal{P} \in L_{p}\left(Q_{\tau}\right)$ as a solution to problem $4.3-4.5$ for $T=\tau$. After that we let $\mathcal{F}_{\tau}[z]=\varphi$. By integral representation (5.5) in 31 we then conclude that Condition $\mathbf{G}_{1}$ ) holds true. Condition $\mathbf{G}_{2}$ ) is satisfied by Lemma 4.1 with $\mathcal{N}(\omega)=\mathcal{N}=C_{1}\left(1+e^{\gamma T}\right), \alpha_{0} \equiv 1$. Condition $\left.\mathbf{G}_{3}\right)$ obviously holds since according Lemma 4.1.

$$
\left\|\mathcal{F}_{\tau}(0)\right\|_{W[0 ; \tau]} \leqslant \mathcal{N}\left\|\varphi_{0}\right\|_{p, \Omega}
$$


Then we consider problem 4.4 - 4.5 for a non-controlled nonlinear Navier-Stokes system

$$
\frac{\partial \varphi}{\partial t}-\nu \Delta \varphi+a(x, t) \varphi+\sum_{k=1}^{3} a_{k}(x, t) \varphi_{x_{k}}+\nabla \mathcal{P}=f[\varphi](x, t),(x, t) \in Q_{T}
$$

where

$$
f[\varphi](x, t)=g(x, t)-\sum_{k=1}^{3} \varphi_{k} \varphi_{x_{k}}, \quad g \in L_{p}\left(Q_{T}\right), \quad p \geqslant \frac{5}{3} .
$$

We are going to obtained needed estimate for the right hand side $f$. At that we shall make use some constructions made in [31, Sect. 10]. We find numbers the $\sigma, q \geqslant 1$ by the conditions:

$$
\begin{aligned}
& \max \left\{\frac{7}{2 p}-\frac{3}{2}, 0\right\} \leqslant \frac{1}{\sigma}<\frac{1}{p}, \\
& 1+\frac{3}{2 p q}-\frac{5}{2 p}>0, \quad \frac{1}{2}+\frac{3}{2 p q^{\prime}}-\frac{5}{2 p}>0,
\end{aligned}
$$

where $q^{\prime}$ is dual to $q$. Let us show that system 4.13), 4.14 is compatible. We take, for instance, $q=2$. Then $q^{\prime}=2$ and condition (4.14 becomes:

$$
\frac{1}{2}+\frac{3}{4 p}-\frac{5}{2 p}>0 \quad \Leftrightarrow \quad 2 p+3-10>0 \quad \Leftrightarrow \quad p>\frac{7}{2} .
$$

Hence,

$$
\frac{7}{2 p}-\frac{3}{2}<1-\frac{3}{2}=-\frac{1}{2}
$$

and 4.13 becomes

$$
0 \leqslant \frac{1}{\sigma}<\frac{1}{p} \quad \Leftrightarrow \quad \sigma>p
$$

According Lemma 4.3, it follows from 4.14 that for $\varphi \in W[0 ; \tau], r \in(1 ; \infty)$ we have:

$$
\varphi \in L_{p q, \sigma r}\left(Q_{\tau}\right), \quad \varphi_{x_{k}} \in L_{p q^{\prime}, \sigma r^{\prime}}\left(Q_{\tau}\right) \text {. }
$$

We denote:

$$
H[\varphi]=H[\varphi, \varphi], \quad H[\varphi, \psi]=\sum_{k=1}^{3} \varphi_{k} \psi_{x_{k}}, \quad \varphi, \psi \in W[0 ; \tau] .
$$

Employing Hölder inequality for $H=H[\varphi, \psi]$, we get:

$$
\|H\|_{p, Q_{\tau}}^{p}=\int_{0}^{\tau} d t \int_{\Omega}|H|^{p} d x \leqslant\left(\int_{0}^{\tau} 1^{\xi^{\prime}} d t\right)^{\frac{1}{\xi^{\prime}}}\left(\int_{0}^{\tau}\left\{\int_{\Omega}|H|^{p} d x\right\}^{\xi}\right)^{\frac{1}{\xi}}
$$

where

$$
\xi=\frac{\sigma}{p} \quad \Rightarrow \quad \xi^{\prime}=\frac{\xi}{\xi-1}=\frac{\sigma}{\sigma-p}
$$

Thus,

$$
\|H\|_{p, Q_{\tau}}^{p} \leqslant \tau^{1-\frac{p}{\sigma}}\left(\int_{0}^{\tau}\|H\|_{p, \Omega}^{\sigma} d t\right)^{\frac{p}{\sigma}}
$$

and this yields

$$
\|H\|_{p, Q_{\tau}} \leqslant \tau^{\frac{1}{p}-\frac{1}{\sigma}}\|H\|_{p, \sigma, Q_{\tau}} \leqslant \tau^{\frac{1}{p}-\frac{1}{\sigma}} \sum_{k=1}^{3}\left\|\varphi_{k} \psi_{x_{k}}\right\|_{p, \sigma, Q_{\tau}}
$$


By the Hölder inequality

$$
\left\|\left(\varphi_{k} \psi_{x_{k}}\right)(\cdot, t)\right\|_{p, \Omega}^{p} \leqslant\left(\int_{\Omega}\left|\varphi_{k}\right|^{p q} d x\right)^{\frac{1}{q}}\left(\int_{\Omega}\left|\psi_{x_{k}}\right|^{p q^{\prime}} d x\right)^{\frac{1}{q^{\prime}}},
$$

and therefore,

$$
\left\|\left(\varphi_{k} \psi_{x_{k}}\right)(\cdot, t)\right\|_{p, \Omega} \leqslant\left\|\varphi_{k}\right\|_{p q, \Omega}\left\|\psi_{x_{k}}\right\|_{p q^{\prime}, \Omega} .
$$

Hence,

$$
\begin{aligned}
\left\|\varphi_{k} \psi_{x_{k}}\right\|_{p, \sigma, Q_{\tau}}^{\sigma} & =\int_{0}^{\tau}\left\|\varphi_{k} \psi_{x_{k}}\right\|_{p, \Omega}^{\sigma} d t \leqslant \int_{0}^{\tau}\left\|\varphi_{k}\right\|_{p q, \Omega}^{\sigma}\left\|\psi_{x_{k}}\right\|_{p q^{\prime}, \Omega}^{\sigma} d t \\
& \leqslant\left(\int_{0}^{\tau}\left\|\varphi_{k}\right\|_{p q, \Omega}^{\sigma r} d t\right)^{\frac{1}{r}}\left(\int_{0}^{\tau}\left\|\psi_{x_{k}}\right\|_{p q^{\prime}, \Omega}^{\sigma r^{\prime}} d t\right)^{\frac{1}{r^{\prime}}} .
\end{aligned}
$$

This implies the following lemma.

Lemma 4.4. For all $\varphi, \psi \in W[0 ; \tau]$ the estimates

$$
\begin{aligned}
& \left\|\varphi_{k} \psi_{x_{k}}\right\|_{p, \sigma, Q_{\tau}} \leqslant\left\|\varphi_{k}\right\|_{p q, \sigma r, Q_{\tau}}\left\|\psi_{x_{k}}\right\|_{p q^{\prime}, \sigma r^{\prime}, Q_{\tau}}, \\
& \|H\|_{p, Q_{\tau}} \leqslant \tau^{\frac{1}{p}-\frac{1}{\sigma}} \sum_{k=1}^{3}\left\|\varphi_{k}\right\|_{p q, \sigma r, Q_{\tau}}\left\|\psi_{x_{k}}\right\|_{p q^{\prime}, \sigma r^{\prime}, Q_{\tau}}
\end{aligned}
$$

hold true.

This lemma implies the next one.

Lemma 4.5. Let $\tau, \xi \in(0 ; T], \tau \leqslant \xi, \varphi, \psi \in W[0 ; \xi]$. Then

$$
\begin{aligned}
& \|H\|_{L_{p}([\tau ; \xi] ; Y)} \leqslant c(\xi-\tau)^{\frac{1}{p}-\frac{1}{\sigma}} \sum_{k=1}^{3}\left\|\psi_{x_{k}}\right\|_{p q^{\prime}, \sigma r^{\prime}, Q_{\tau}}\left\{\|\varphi\|_{W[0 ; \tau]}+\|\varphi(\cdot, 0)\|_{p, \Omega}\right\}, \\
& \|H\|_{L_{p}([\tau ; \xi] ; Y)} \leqslant c(\xi-\tau)^{\frac{1}{p}-\frac{1}{\sigma}} \sum_{k=1}^{3}\left\|\varphi_{k}\right\|_{p q, \sigma r, Q_{\tau}}\left\{\|\psi\|_{W[0 ; \tau]}+\|\psi(\cdot, 0)\|_{p, \Omega}\right\} .
\end{aligned}
$$

We consider the difference

$$
H[\varphi]-H[\psi]=\sum_{k=1}^{3}\left(\varphi_{k} \varphi_{x_{k}}-\psi_{k} \psi_{x_{k}}\right)=H[\varphi-\psi, \varphi]+H[\psi, \varphi-\psi] .
$$

Thus, Lemmata 4.3, 4.5 imply the following statement.

Lemma 4.6. Let $0 \leqslant \tau \leqslant \xi \leqslant T, \varphi, \psi \in W_{0}[0 ; \xi]$,

$$
\|\varphi\|_{W[0 ; \xi]} \leqslant M, \quad\|\psi\|_{W[0 ; \xi]} \leqslant M
$$

Then

$$
\|H[\varphi]-H[\psi]\|_{L_{p}([\tau ; \xi] ; Y)} \leqslant \alpha_{1}(\xi-\tau) \beta_{1}(M)\|\varphi-\psi\|_{W[0 ; \tau]},
$$

where $\alpha_{1}(\delta)=\delta^{\frac{1}{p}-\frac{1}{\sigma}}, \beta_{1}(M)=3 c^{2}\left(M+\left\|\varphi_{0}\right\|_{p, \Omega}\right)$.

Remark 4.2. Inequality 4.17) in the formulation of Lemma 4.6 means that $H[\varphi]$ satisfies Condition $\mathbf{F}_{2}$ ). Condition $\left.\mathbf{F}_{1}\right)$ is obviously true. Condition $\mathbf{F}_{3}$ ) is satisfied thanks to Lemma 2.1. 
Now we consider a controlled operator

$$
g=g[u](\varphi)(x, t), \quad u \in U,
$$

satisfying Conditions $\mathbf{F}_{1}$ ) $-\mathbf{F}_{2}$ ) with the parameters $\alpha_{1}=\alpha_{g}, \beta_{1}=\beta_{g}$. In particular, from a physical point of view, it is of interest to control the density of external fields by the feedback principle with a linear pattern: $g=u_{1}(x, t)+u_{2}(x, t) \varphi(x, t)$. A corresponding problem can be treated as a problem with a program control $u=\left(u_{1}, u_{2}\right)$. It is clear that in the case $u_{1} \in L_{p}\left(Q_{T}\right), u_{2} \in L_{p q^{\prime}, \sigma r^{\prime}}\left(Q_{T}\right)$ we can obtain estimates of the same type as above for the function $H[\varphi]$.

We consider the operator of the right hand side: $f[u](\varphi)=g[u](\varphi)-H[\varphi]$. It is clear $f[u]$ satisfies Conditions $\left.\mathbf{F}_{1}\right)-\mathbf{F}_{2}$ ). Condition $\mathbf{F}_{3}$ ) thanks to Lemma 2.1 .

We consider problem 4.4 - 4.5 for a controlled nonlinear Naviers-Stokes system:

$$
\frac{\partial \varphi}{\partial t}-\nu \Delta \varphi+a(x, t) \varphi+\sum_{k=1}^{3} a_{k}(x, t) \varphi_{x_{k}}+\nabla \mathcal{P}=f[u](\varphi)(x, t), \quad(x, t) \in Q_{T} .
$$

We have thus established that problem 4.4 - 4.5 for equation 4.18 possesses SEGS property.

We note that in [31, Sect. 10], the unique solvability of problem (4.4) - 4.5) for a noncontrolled equation of form 4.12 was proved only under the assumption that the final time $T$ is small enough.

In general, except for some particular cases like absence of forces $g=0$, axial symmetry, etc., and even in these particular cases, the issues of global solvability and those of uniqueness of the solutions to non-linear non-stationary Navier-Stokes systems were studied mostly independently in various spaces, see, for instance, [32], |33], [34], |35], [36], |37], |38], [39]. To find one class, in which it would be possible to prove the global stability and uniqueness of the solution to this system in a general case is still a topical problem.

Finally we mention the results in $[3$, Ch. 3, Sects. 4-5; Ch. 4], where problem (4.4)-(4.5) was considered for equation of form (4.12) as $a=0, a_{k}=0, k=\overline{1,3}$, and the function $g(t, x)$ was regarded as a control in a problem on minimizing the energy in speeding up a stationary $\varphi_{0}=0$ liquid up to a prescribed velocity. It was proved that the set of the controls for which there exists a unique global solution (for an arbitrarily fixed $T>0$ ) is everywhere dense in the space of the right hand sides. It was also observed that the uniqueness of the solution can be proved only in a class of sufficiently smooth functions, for instance, $W[0 ; T]$. However, the solvability in this class for arbitrary $T>0$ was not proved since the problem was ill-posed.

\section{ExAmple: Benjamin-Bona-Mahony-Burgers EQUATION}

Let $\Omega \subset \mathbb{R}^{\ell}$ be a bounded domain with a boundary $\partial \Omega$. Following $[1]$, we introduce a series of notations.

$\mathbb{H}_{0}^{m}(\Omega)$ is a Hilbert space of measurable functions having the zero trace on $\partial \Omega$, possessing generalized derivatives of orders up to $m, m \in \mathbb{N}$, in the space $L_{2}(\Omega)$. The scalar product in $\mathbb{H}_{0}^{m}(\Omega)$ is

$$
(\varphi, \psi)_{\mathbb{H}_{0}^{m}}=\sum_{|\mu| \leqslant m}\left(\partial^{\mu} \varphi, \partial^{\mu} \psi\right)_{2}
$$

where $(\cdot, \cdot)_{2}$ is the scalar product in $L_{2}(\Omega)$;

$\mathbb{H}^{-m}(\Omega)$ is the Hilbert space dual to $\mathbb{H}_{0}^{m}(\Omega)$. Each element of this dual space can be represented as

$$
\varphi=\sum_{|\mu| \leqslant m} \partial^{\mu} g_{\mu}, \quad g_{\mu} \in L_{2}(\Omega)
$$

see [11]. Here we mean that $\varphi$ is a linear continuous functional on $\mathbb{H}_{0}^{m}(\Omega)$ and its action on an element $\psi \in \mathbb{H}_{0}^{m}(\Omega)$, denoted by $\langle\varphi, \psi\rangle$, is called duality bracket between the spaces $\mathbb{H}_{0}^{m}(\Omega)$ 
and $\mathbb{H}^{-m}(\Omega)$. It is calculated by the formula:

$$
\langle\varphi, \psi\rangle=\sum_{|\mu| \leqslant m}(-1)^{|\mu|}\left(g_{\mu}, \partial^{\mu} \psi\right)_{2}=\sum_{|\mu| \leqslant m}(-1)^{|\mu|} \int_{\Omega} g_{\mu} \partial^{\mu} \psi d x
$$

for more details, see [40, Subsect. 1.2.16].

$\mathbb{W}^{k, p}(\Omega)$ is a Banach space of measurable functions having all generalized derivatives of order up to $k$ in the space $L_{p}(\Omega), p \geqslant 1$. The norm in the introduced space reads as

$$
\|\varphi\|_{k, p}=\sum_{|\mu| \leqslant k}\left\|\partial^{\mu} \varphi\right\|_{p}
$$

where $\|\cdot\|_{p}$ is the norm in $L_{p}(\Omega)$;

$\mathbb{W}_{0}^{k, p}(\Omega)$ is the Banach space of elements in $\mathbb{W}^{k, p}(\Omega)$ having zero trace on $\partial \Omega$;

$\mathbb{W}^{-k, p^{\prime}}(\Omega)$ is the Banach space dual to $\mathbb{W}_{0}^{k, p}(\Omega), p^{\prime}=\frac{p}{p-1}$, whose elements can be represented as

$$
\varphi=\sum_{|\mu| \leqslant k} \partial^{\mu} g_{\mu}, \quad g_{\mu} \in L_{p^{\prime}}(\Omega)
$$

$\partial \Omega \in C^{(m, \delta)}$ is the boundary of the domain $\Omega$, and in a vicinity of each point $x \in \partial \Omega$ it can be represented by local coordinates

$$
\xi_{i}=\Phi_{i}\left(\xi_{1}, \ldots, \xi_{\ell-1}, \eta\right), \quad i=\overline{1, \ell-1}
$$

and the functions $\Phi_{i}$ are $m$ times continuously differentiable in all variables and $\Phi_{i}^{(m)}, m \in \mathbb{Z}_{+}^{\ell}$, are Hölder functions with an exponent $\delta \in(0 ; 1]$.

We let $Y=\mathbb{H}_{0}^{1}(\Omega), Y^{-}=\mathbb{H}^{-1}(\Omega)$. Following [11, Sect. 11], we begin with considering Dirichlet initial boundary value problem for a three-dimensional Benjamin-Bona-MahonyBurgers equation (BBMBE):

$$
\begin{aligned}
& \frac{\partial}{\partial t}(\Delta \varphi-\varphi)+\Delta \varphi+\varphi \varphi_{x_{1}}+\varphi^{3}=0, \quad(x, t) \in Q_{T}=\Omega \times(0 ; T], \\
& \left.\varphi(x, t)\right|_{\partial \Omega}=0 ; \quad \varphi(x, 0)=\varphi_{0}(x), \quad x \in \Omega,
\end{aligned}
$$

where $x=\left(x_{1}, x_{2}, x_{3}\right) \in \Omega \subset \mathbb{R}^{3}, \partial \Omega \in C^{(2, \delta)}, \delta \in(0 ; 1]$. As it was said in [11, Sect. 11], such problem arises in studying non-stationary processes in semiconductors under the presence of sources and an external homogeneous constant electric field. A strong generalized equation of problem (5.1), 5.2) is defined as a function in the class $C^{(1)}([0 ; T] ; Y)$ obeying the conditions:

$$
\begin{aligned}
& \left\langle\Delta \varphi^{\prime}-\varphi^{\prime}+\Delta \varphi+\varphi \varphi_{x_{1}}+\varphi^{3}, w\right\rangle=0 \quad \text { for all } \quad w \in Y, \quad t \in[0 ; T] \\
& \varphi(0)=\varphi_{0} \in Y,
\end{aligned}
$$

where $\langle\cdot, \cdot\rangle$ is the dual bracketing between the spaces $Y$ and $Y^{-}$. The operator $A_{1}=-\Delta \varphi$ : $Y \rightarrow Y^{-}$is treated in the following sense:

$$
\left\langle A_{1} \varphi, \psi\right\rangle=(\nabla \varphi, \nabla \psi)_{2} \quad \text { for all } \quad \varphi, \psi \in Y .
$$

Following [11, we denote

$$
A \varphi=-\Delta \varphi+\varphi, \quad F_{1}(\varphi)=\frac{1}{2} \frac{\partial \varphi^{2}}{\partial x_{1}}, \quad F_{2}(\varphi)=\varphi^{3}, \quad F(\varphi)=\varphi+F_{1}(\varphi)+F_{2}(\varphi),
$$

and we reduce problem (5.1), (5.2) to an abstract Cauchy problem for an operator differential equation:

$$
A \frac{d \varphi}{d t}+A \varphi=F(\varphi), \quad \varphi(0)=\varphi_{0} \in Y
$$


As it was mentioned in [11, Sect. 11], the operator $A: Y \rightarrow Y^{-}$possesses an inverse Lipschitzcontinuous operator:

$$
\left\|A^{-1} z_{1}-A^{-1} z_{2}\right\|_{Y} \leqslant\left\|z_{1}-z_{2}\right\|_{Y^{-}} \quad \text { for all } z_{1}, z_{2} \in Y^{-} .
$$

Moreover, this Cauchy problem is equivalent to an abstract integral equation:

$$
\varphi(t)=\varphi_{0} e^{-t}+\int_{0}^{t} d s e^{-(t-s)} A^{-1} F(\varphi), \quad \varphi \in L_{\infty}([0 ; T] ; Y) .
$$

We consider a corresponding equation with a fixed right hand side $z \in L_{\infty}([0 ; T] ; Y)$ :

$$
\varphi(t)=\varphi_{0} e^{-t}+\int_{0}^{t} d s e^{-(t-s)} z(s), \quad \varphi \in L_{\infty}([0 ; T] ; Y) .
$$

It is clear that

$$
\|\varphi\|_{L_{\infty}([0 ; \tau] ; Y)} \leqslant\left\|\varphi_{0}\right\|_{Y}+\int_{0}^{\tau}\|z(s)\|_{Y} d s
$$

for each $\tau \in(0 ; T]$. For each $\tau \in(0 ; T]$, formula (5.4) defines an operator

$$
\mathcal{F}_{\tau}: W[0 ; \tau] \rightarrow W_{0}[0 ; \tau]
$$

where we formally adopt the notation

$$
W_{0}[0 ; \tau]=W[0 ; \tau]=L_{\infty}([0 ; \tau] ; Y) .
$$

Condition $\mathbf{G}_{1}$ ) obviously holds, see (5.4). If $z_{1}, z_{2} \in W[0 ; \xi],\left.z_{1}\right|_{[0 ; \tau]}=\left.z_{2}\right|_{[0 ; \tau]}$, the by (5.4), (5.5), we get:

$$
\left\|\mathcal{F}_{\xi}\left[z_{1}\right]-\mathcal{F}_{\xi}\left[z_{2}\right]\right\|_{W[0 ; \xi]} \leqslant \int_{\tau}^{\xi}\left\|z_{1}(s)-z_{2}(s)\right\|_{Y} d s \leqslant(\xi-\tau)\left\|z_{1}-z_{2}\right\|_{L_{\infty}([\tau ; \xi] ; Y)} .
$$

This means that Condition $\mathbf{G}_{2}$ ) holds for $\mathcal{N} \equiv 1, \alpha_{0}(t)=t$. Condition $\mathbf{G}_{3}$ ) holds as well since $\mathcal{F}_{\tau}(0)=\varphi_{0}$.

We define an operator $G(\varphi)=A^{-1} F(\varphi)$. According [11, Sect. 11], $G(\varphi)$ can be regarded as an operator $G: W[0 ; \tau] \rightarrow W[0 ; \tau]$, and moreover, the estimates hold:

$$
\begin{array}{llll}
\left\|F\left(\varphi_{1}\right)-F\left(\varphi_{2}\right)\right\|_{Y^{-}} \leqslant \mu(M)\left\|\varphi_{1}-\varphi_{2}\right\|_{Y} & \text { for all } \quad \varphi_{i} \in Y, & \left\|\varphi_{i}\right\|_{Y} \leqslant M, & i=1,2 ; \\
\left\|G\left(\varphi_{1}\right)-G\left(\varphi_{2}\right)\right\|_{Y} \leqslant \mu(M)\left\|\varphi_{1}-\varphi_{2}\right\|_{Y} & \text { for all } \quad \varphi_{i} \in Y, \quad\left\|\varphi_{i}\right\|_{Y} \leqslant M, & i=1,2 .
\end{array}
$$

Hence,

$$
\left\|G\left(\varphi_{1}\right)-G\left(\varphi_{2}\right)\right\|_{L_{\infty}([\tau ; \xi] ; Y)} \leqslant \mu(M)\left\|\varphi_{1}-\varphi_{2}\right\|_{W[0 ; \tau]}
$$

for all $\varphi_{i} \in W[0 ; \tau],\left\|\varphi_{i}\right\|_{W[0 ; \tau]} \leqslant M, i=1,2$. Thus, the operator $G$ satisfies Condition $\mathbf{F}_{2}$ ) as $\alpha_{1} \equiv 1, \beta_{1}(M)=\mu(M)$. Condition $\mathbf{F}_{1}$ ) obviously holds since there is no explicit dependence on the variable $t$ in the right hand side except for an implicit dependence in the argument $\varphi$. Condition $\mathbf{F}_{3}$ ) is implied directly by Lemma 2.1. Equation (5.3) is equivalent to the equation

$$
\varphi=\mathcal{F}_{T}[G(\varphi)], \quad \varphi \in W[0 ; T] .
$$

As it was shown, all conditions $\left.\left.\left.\mathbf{G}_{1}\right)-\mathbf{G}_{3}\right), \mathbf{F}_{1}\right)-\mathbf{F}_{3}$ ) are satisfied. We note that [11, Sect. 11]

$$
\begin{array}{ll}
\mathcal{F}_{\tau}: L_{\infty}([0 ; \tau] ; Y) \rightarrow \mathbb{A}([0 ; \tau] ; Y), & \mathcal{F}_{\tau}: C([0 ; \tau] ; Y) \rightarrow C^{(1)}([0 ; \tau] ; Y), \\
\mathcal{F}_{\tau} G: L_{\infty}([0 ; \tau] ; Y) \rightarrow \mathbb{A} \mathbb{C}([0 ; \tau] ; Y), & \mathcal{F}_{\tau} G: \mathbb{A} \mathbb{C}([0 ; \tau] ; Y) \rightarrow C^{(1)}([0 ; \tau] ; Y) .
\end{array}
$$

This is why the solution in fact belongs to the class $C^{(1)}([0 ; \tau] ; Y)$. 
We consider a corresponding controlled analogue:

$$
A \frac{d \varphi}{d t}+A \varphi=F(\varphi)+g[u](\varphi), \quad \varphi \in C^{(1)}([0 ; T] ; Y)
$$

which is equivalent to the equation

$$
\varphi=\mathcal{F}_{T}[f[u](\varphi)], \quad \varphi \in W[0 ; T]
$$

where $f[u](\varphi)=A^{-1}[F(\varphi)+g[u](\varphi)]$. We assume that

$$
\begin{aligned}
& g[u]: Y \rightarrow Y^{-}, \quad g[u]: L_{\infty}([0 ; \tau] ; Y) \rightarrow L_{\infty}\left([0 ; \tau] ; Y^{-}\right), \quad \tau \in(0 ; T], \\
& g[u]: \mathbb{A} \mathbb{C}([0 ; T] ; Y) \rightarrow \mathbb{A} \mathbb{C}\left([0 ; T] ; Y^{-}\right), \quad u \in U, \\
& \left\|g[u]\left(\varphi_{1}\right)-g[u]\left(\varphi_{2}\right)\right\|_{Y^{-}} \leqslant \mu_{g}(M) \quad \text { for all } \quad \varphi_{i} \in Y, \quad\left\|\varphi_{i}\right\|_{Y} \leqslant M, \quad i=1,2 .
\end{aligned}
$$

Then the operator $f[u](\varphi)$ satisfy Conditions $\left.\left.\mathbf{F}_{1}\right)-\mathbf{F}_{3}\right)$ in the same way as for $G(\varphi), \alpha_{1} \equiv 1$, $\beta_{1}(M)=\mu(M)+\mu_{g}(M)$. Thus, according Theorem 2.2, the SEGS property holds.

We note that in [11], a local solvability and the existence of a maximal solution were proved for problem (5.1), (5.2). There was also provided a way for finding a segment $\left[T_{1} ; T_{2}\right]$ such that as $T \in\left(0 ; T_{1}\right)$, there exists a unique global solution, while for $T \geqslant T_{2}$ the solution blows up.

\section{EXAMPLE: STRONGLY NONLINEAR PSEUDO-PARABOLIC EQUATIONS}

Let $\Omega \subset \mathbb{R}^{3}$ be a surface simply connected bounded domain. Following [11, Subsect. 8.1], we consider initial boundary value problems for strongly nonlinear pseudo-parabolic equations of form

$$
\begin{aligned}
& \left\{\begin{array}{l}
\frac{\partial}{\partial t}(\Delta \varphi-\varphi)+\varphi \frac{\partial \varphi}{\partial x_{1}}+\varphi^{3}=0 ; \\
\left.\varphi\right|_{\partial \Omega}=0, \quad t \in[0 ; T] ; \quad \varphi(x, 0)=\varphi_{0}(x), \quad x \in \Omega ; \quad \partial \Omega \in C^{(2, \delta)} ;
\end{array}\right. \\
& \left\{\begin{array}{l}
\frac{\partial}{\partial t}\left(-\Delta^{2} \varphi+\Delta \varphi\right)+\varphi \frac{\partial \varphi}{\partial x_{1}}-\operatorname{div}\left(|\nabla \varphi|^{2} \nabla \varphi\right)=0 ; \\
\left.\varphi\right|_{\partial \Omega}=\left.\frac{\partial \varphi}{\partial \mathbf{n}}\right|_{\partial \Omega}=0, \quad t \in[0 ; T] ; \quad \varphi(x, 0)=\varphi_{0}(x), \quad x \in \Omega ; \quad \partial \Omega \in C^{(4, \delta)} ;
\end{array}\right. \\
& \left\{\begin{array}{l}
\frac{\partial}{\partial t}\left(-\Delta^{2} \varphi+\Delta \varphi\right)+\sum_{i=1}^{3} b_{i} \frac{\partial}{\partial x_{i}} \prod_{j \neq i} \frac{\partial \varphi}{\partial x_{j}}-\operatorname{div}\left(|\nabla \varphi|^{2} \nabla \varphi\right)=0 ; \\
\left.\varphi\right|_{\partial \Omega}=\left.\frac{\partial \varphi}{\partial \mathbf{n}}\right|_{\partial \Omega}=0, \quad t \in[0 ; T] ; \quad \varphi(x, 0)=\varphi_{0}(x), \quad x \in \Omega ; \quad \partial \Omega \in C^{(4, \delta)} ;
\end{array}\right.
\end{aligned}
$$

in a cylinder $Q_{T}=\Omega \times(0 ; T] \ni(x, t)$, where $b_{1}+b_{2}+b_{3}=0, \delta \in(0 ; 1], x=\left(x_{1}, x_{2}, x_{3}\right) \in \Omega$.

In [11, Subsect. 8.1], problems (6.1) - (6.3) were rewritten as the following Cauchy problems for abstract first order differential equations with operator coefficients, respectively:

$$
\begin{array}{ll}
A_{1} \frac{d \varphi}{d t}=F_{3}(\varphi)-F_{1}(\varphi), & \varphi(0)=\varphi_{0} \in \mathbb{H}_{0}^{1}(\Omega), \\
A_{2} \frac{d \varphi}{d t}=F_{4}(\varphi)-F_{1}(\varphi), & \varphi(0)=\varphi_{0} \in \mathbb{H}_{0}^{2}(\Omega), \\
A_{2} \frac{d \varphi}{d t}=F_{4}(\varphi)-F_{2}(\varphi), & \varphi(0)=\varphi_{0} \in \mathbb{H}_{0}^{2}(\Omega)
\end{array}
$$


Here

$$
\begin{aligned}
& A_{1} \varphi=-\Delta \varphi: \mathbb{H}_{0}^{1}(\Omega) \rightarrow \mathbb{H}^{-1}(\Omega), \quad A_{2} \varphi=\Delta^{2} \varphi-\Delta \varphi: \mathbb{H}_{0}^{2}(\Omega) \rightarrow \mathbb{H}^{-2}(\Omega), \\
& \left\langle A_{1} \varphi, \psi\right\rangle_{1}=(\nabla \varphi, \nabla \psi)_{2}=\int_{\Omega} \nabla \varphi \cdot \nabla \psi d x \\
& \left\langle A_{2} \varphi, \psi\right\rangle_{2}=(\nabla \varphi, \nabla \psi)_{2}+(\Delta \varphi, \Delta \psi)_{2}=\int_{\Omega}[\nabla \varphi \cdot \nabla \psi+\Delta \varphi \Delta \psi] d x,
\end{aligned}
$$

$\langle\cdot, \cdot\rangle_{m}$ is the duality bracketing between $\mathbb{H}_{0}^{m}(\Omega)$ and $\mathbb{H}^{-m}(\Omega),(\cdot, \cdot)_{p}$ is the duality bracketing between $L_{p}(\Omega)$ and $L_{p^{\prime}}(\Omega), p^{\prime}=\frac{p}{p-1}$;

$$
\begin{aligned}
& F_{1}(\varphi)=\frac{1}{2} \frac{\partial \varphi^{2}}{\partial x_{1}}: \mathbb{H}_{0}^{1}(\Omega) \subset L_{4}(\Omega) \rightarrow \mathbb{H}^{-1}(\Omega), \\
& F_{2}(\varphi)=\sum_{i=1}^{3} b_{i} \frac{\partial}{\partial x_{i}} \prod_{j \neq i} \frac{\partial \varphi}{\partial x_{j}}: \mathbb{H}_{0}^{2}(\Omega) \rightarrow \mathbb{H}^{-2}(\Omega), \\
& F_{3}(\varphi)=\varphi^{3}: \mathbb{H}_{0}^{1}(\Omega) \subset L_{4}(\Omega) \rightarrow L_{4 / 3}(\Omega) \subset \mathbb{H}^{-1}(\Omega), \\
& F_{4}(\varphi)=-\operatorname{div}\left(|\nabla \varphi|^{2} \nabla \varphi\right): \mathbb{H}_{0}^{2}(\Omega) \subset \mathbb{W}_{0}^{1,4}(\Omega) \rightarrow \mathbb{W}^{-1,4 / 3}(\Omega) \subset \mathbb{H}^{-2}(\Omega) .
\end{aligned}
$$

Then in [11, Subsect. 8.1] each of problems (6.4) - (6.6) was rewritten as the Cauchy problem for an abstract ordinary differential equations in the Banach space $Y$ :

$$
A \frac{d \varphi}{d t}=F(\varphi), \quad \varphi(0)=\varphi_{0} \in Y
$$

where $A: Y \rightarrow Y^{*}, F: Y \rightarrow Y^{*}$, and, as it was proved in [11, Subsect. 8.1],

$$
\begin{aligned}
& \left\|A \varphi_{1}-A \varphi_{2}\right\|_{Y^{*}} \geqslant m\left\|\varphi_{1}-\varphi_{2}\right\|_{Y}, \quad \text { for all } \quad \varphi_{i} \in Y, \quad i=1,2, \\
& \left\|F\left(\varphi_{1}\right)-F\left(\varphi_{2}\right)\right\|_{Y^{*}} \leqslant \mu(M)\left\|\varphi_{1}-\varphi_{2}\right\|_{Y} \quad \text { for all } \quad \varphi_{i} \in Y, \quad\left\|\varphi_{i}\right\|_{Y} \leqslant M, \quad i=1,2,
\end{aligned}
$$

and the operator $A$ possesses an inverse Lipschitz-continuous operator $A^{-1}: Y^{*} \rightarrow Y$ :

$$
\left\|A^{-1} z_{1}-A^{-1} z_{2}\right\|_{Y} \leqslant m^{-1}\left\|z_{1}-z_{2}\right\|_{Y^{*}} \quad \text { for all } z_{1}, z_{2} \in Y^{*}
$$

For (6.4) we choose $Y=\mathbb{H}_{0}^{1}(\Omega)$, while for $(6.5)$ and $(6.6)$ a similar choice is $Y=\mathbb{H}_{0}^{2}(\Omega)$. As it was shown in the above cited work, problem (6.7) is equivalent to the following one:

$$
\frac{d \varphi}{d t}=G(\varphi)(t), \quad t \in(0 ; T] ; \quad \varphi(0)=\varphi_{0} \in Y ; \quad \varphi \in C^{(1)}([0 ; T] ; Y),
$$

where $G(\varphi)=A^{-1} F(\varphi): C([0 ; T] ; Y) \rightarrow C([0 ; T] ; Y)$. Finally, problem $[6.8$ is equivalent to an abstract integral equation:

$$
\varphi(t)=\varphi_{0}+\int_{0}^{t} d s G(\varphi)(s), \quad t \in[0 ; T] ; \quad \varphi \in C([0 ; T] ; Y) .
$$

For an arbitrary $\tau \in(0 ; T]$, we consider a following analogue with a fixed right hand side $z \in C([0 ; \tau] ; Y):$

$$
\varphi(t)=\varphi_{0}+\int_{0}^{t} z(s) d s, \quad t \in[0 ; \tau] ; \quad \varphi \in C([0 ; \tau] ; Y) .
$$

Formula 6.10 defines an operator $\mathcal{F}_{\tau}: C([0 ; \tau] ; Y) \rightarrow W[0 ; \tau], \varphi=\mathcal{F}_{\tau}[z]$, where $W[0 ; \tau]=$ $C([0 ; \tau] ; Y)$. As $W_{0}[0 ; \tau]$, we can take the set of all $\varphi \in W[0 ; \tau]$ such that $\varphi(0)=\varphi_{0}$. 
Condition $\left.\mathbf{G}_{1}\right)$ is obviously satisfied, see 6.10 . If $z_{1}, z_{2} \in W[0 ; \xi],\left.z_{1}\right|_{[0 ; \tau]}=\left.z_{2}\right|_{[0 ; \tau]}$, then by 6.10 we obtain:

$$
\left\|\mathcal{F}_{\xi}\left[z_{1}\right]-\mathcal{F}_{\xi}\left[z_{2}\right]\right\|_{W[0 ; \xi]} \leqslant \int_{\tau}^{\xi}\left\|z_{1}(s)-z_{2}(s)\right\|_{Y} d s \leqslant(\xi-\tau)\left\|z_{1}-z_{2}\right\|_{C([\tau ; \xi] ; Y)} .
$$

This means that Condition $\mathbf{G}_{2}$ ) holds as $\mathcal{N} \equiv 1, \alpha_{0}(t)=t$. Condition $\left.\mathbf{G}_{3}\right)$ is satisfied as well since $\mathcal{F}_{\tau}(0)=\varphi_{0}$.

The operator $G(\varphi)$ obviously satisfies Condition $\mathbf{F}_{1}$ ) since the formula defining this operator involves no explicit time dependence. Let

$$
\varphi_{i} \in W[0 ; \xi], \quad\left\|\varphi_{i}\right\|_{C([0 ; \xi] ; Y)} \leqslant M, \quad i=1,2 .
$$

Then

$$
\begin{aligned}
\left\|G\left(\varphi_{1}\right)(t)-G\left(\varphi_{2}\right)(t)\right\|_{Y} & \leqslant m^{-1}\left\|F\left(\varphi_{1}\right)(t)-F\left(\varphi_{2}\right)(t)\right\|_{Y^{*}} \\
& \leqslant m^{-1} \mu(M)\left\|\varphi_{1}(t)-\varphi_{2}(t)\right\|_{Y} \quad \text { for all } \quad t \in[0 ; \xi] .
\end{aligned}
$$

This implies that

$$
\left\|G\left(\varphi_{1}\right)-G\left(\varphi_{2}\right)\right\|_{C([\tau ; \xi] ; Y)} \leqslant m^{-1} \mu(M)\left\|\varphi_{1}-\varphi_{2}\right\|_{C([\tau ; \xi] ; Y)} .
$$

Thus, Condition $\mathbf{F}_{2}$ ) holds as $\alpha_{1} \equiv 1, \beta_{1}(M)=m^{-1} \mu(M)$. Condition $\left.\mathbf{F}_{3}\right)$ is implied by Lemma 2.2.

We consider a following controlled analogue:

$$
A \frac{d \varphi}{d t}=F(\varphi)+g[u](\varphi), \quad \varphi \in C^{(1)}([0 ; T] ; Y)
$$

which is equivalent to the equation

$$
\varphi=\mathcal{F}_{T}[f[u](\varphi)], \quad \varphi \in W[0 ; T],
$$

where $f[u](\varphi)=A^{-1}[F(\varphi)+g[u](\varphi)]$. We assume that

$$
\begin{aligned}
& g[u]: Y \rightarrow Y^{*}, \quad g[u]: C([0 ; \tau] ; Y) \rightarrow C\left([0 ; \tau] ; Y^{*}\right), \quad \tau \in(0 ; T], \quad u \in U, \\
& \left\|g[u]\left(\varphi_{1}\right)-g[u]\left(\varphi_{2}\right)\right\|_{Y^{*}} \leqslant \mu_{g}(M) \quad \text { for all } \quad \varphi_{i} \in Y, \quad\left\|\varphi_{i}\right\|_{Y} \leqslant M, \quad i=1,2 .
\end{aligned}
$$

Then the operator $f[u](\varphi)$ satisfies Conditions $\left.\left.\mathbf{F}_{1}\right)-\mathbf{F}_{3}\right)$ in the same way as for $G(\varphi), \alpha_{1} \equiv 1$, $\beta_{1}(M)=m^{-1}\left(\mu(M)+\mu_{g}(M)\right)$. Hence, according Theorem 2.2 the SEGS property holds.

We note that in [11, Sect. 8], a local solvability and the existence of a maximal solution were proved for problem (6.1) - 6.3). There was also provided a way for finding a segment $\left[T_{1} ; T_{2}\right]$ such that as $T \in\left(0 ; T_{1}\right)$, there exists a unique global solution, while for $T \geqslant T_{2}$ the solution blows up.

\section{BIBLIOGRAPHY}

1. H. Gajewski, K. Gröger, and K. Zacharias. Nichtlineare Operatorgleichungen und Operatordifferentialgleichungen. Akademie-Verlag, Berlin, (1974). (in German).

2. J.L. Lions. Contrôle des systèmes distribués singuliers. Gauthier-Villars, Bordas, Paris (1983). (in French).

3. A.V. Fursikov. Optimal control of distributed systems. Theory and applications. Nauchnaya Kniga, Novosibirsk (1999). [Transl. Math. Monog. 187. Amer. Math. Soc., Providence, RI (2000).]

4. V.I. Sumin. Functional Volterra equations in mathematical theory of optimal control of distributed systems. Habilitation Thesis, Nizhni Novgorod State Univ., Nizhni Novgorod (1998). (in Russian).

5. A.V. Chernov. Volterra operator equations and their applications in optimisation theory of hyperbolic systems. PhD Thesis, Nizhni Novgorod State Univ., Nizhni Novgorod (2000). (in Russian). 
6. V.I. Sumin. The features of gradient methods for distributed optimal control problems // Zhurn. Vychisl. Matem. Matem. Fiz. 30:1, 3-21 (1990). [USSR Comput. Math. Math. Phys. 30:1, 1-15 (1990).]

7. A.V. Chernov. Smooth finite-dimensional approximations of distributed optimization problems via control discretization // Zhurn. Vychisl. Matem. Matem. Fiz. 53:12, 2029-2043 (2013). [Comput. Math. Math. Phys. 53:12, 1839-1852 (2013).]

8. V.K. Kalantarov, O.A. Ladyzhenskaya. The occurrence of collapse for quasilinear equations of parabolic and hyperbolic types // Zapis. Nauchn. Semin. POMI. 69, 77-102 (1977). [J. Soviet Math. 10:1, 53-70 (1978).]

9. V.I. Sumin. Functional Volterra equations in theory of optimal control of distributed systems. Part I. Nizhni Novgorod State Univ., Nizhni Novgorod (1992). (in Russian).

10. O.A. Ladyzhenskaya, N.N. Uraltseva. Linear and quasilinear elliptic equations. Nauka, Moscow (1973). [Academic Press, New York (1987).]

11. M.O. Korpusov, A.G. Sveshnikov. Blow-up of solutions to strongly nonlinear equations of the pseudoparabolic type // Sovrem. Matem. Pril. 40, 3-138 (2006). (in Russian).

12. F. Tröltzsch. Optimal control of partial differential equations: theory, methods and applications. Graduate Studies in Mathematics. 112. Amer. Math. Soc., Providence, RI (2010).

13. C. Carasso, E.H. Hassnaoui. Mathematical analysis of the model arising in study of chemical reactions in a catalytic cracking reactor // Math. Comput. Model. 18: 2, 93-109 (1993).

14. T. Kobayashi, H. Pecher, Y. Shibata. On a global in time existence theorem of smooth solutions to a nonlinear wave equation with viscosity // Math. Ann. 296: 2, 215-234 (1993).

15. P. Biler, D. Hilhorst, T. Nadzieja. Existence and nonexistence of solutions for a model of gravitational interaction of particles. II // Colloq. Math. 67: 2, 297-308 (1994).

16. B. Hu, H.-M. Yin. Global solutions and quenching to a class of quasilinear parabolic equations // Forum Math. 6: 3, 371-383 (1994).

17. G. Lu. Global existence and blow-up for a class of semilinear parabolic systems: a Cauchy problem // Nonlinear Anal. Theory Methods Appl. 24: 8, 1193-1206 (1995).

18. T. Yamazaki. Scattering for a quasilinear hyperbolic equation of Kirchhoff type // J. Diff. Equat. 143: 1, 1-59 (1998).

19. F. Catalano. The nonlinear Klein-Gordon equation with mass decreasing to zero // Adv. Dif. Equat. 7: 9, 1025-1044 (2002).

20. M.M. Cavalcanti, V.N. Domingos Cavalcanti, J.A. Soriano On existence and asymptotic stability of solutions of the degenerate wave equation with nonlinear boundary conditions // J. Math. Anal. Appl. 281: 1, 108-124 (2003).

21. A. Rozanova-Pierrat. Qualitative analysis of the Khokhlov-Zabolotskaya-Kuznetsov (KZK) equation // Math. Models Meth. Appl. Sci. 18:5, 781-812 (2008).

22. X. Zhao. Self-similar solutions to a generalized Davey-Stewartson system // Math. Comput. Model. 50: 9-10, 1394-1399 (2009).

23. M.O. Korpusov, A.V. Ovchinnikov, A.G. Sveshnikov. On blow up of generalized KolmogorovPetrovskii-Piskunov equation // Nonl. Anal., Theory Meth. Appl., Ser. A. Theory Meth. 71: 11, 5724-5732 (2009).

24. A.M. Blokhin, D.L. Tkachev. Asymptotic stability of the stationary solution for a new mathematical model of charge transport in semiconductors // Quat. Appl. Math. 70: 2, 357-382 (2012).

25. H. Saito. Global solvability of the Navier-Stokes equations with a free surface in the maximal regularity $L_{p}-L_{q}$ class // J. Diff. Equat. 264: 3, 1475-1520 (2018).

26. V.I. Sumin. Stability problem for the existence of global solutions to boundary value control problems and Volterra functional equations // Vestn. Nizhegorod. Univ. N. I. Lobachevskogo, Mat. 1, 91107 (2003). (in Russian).

27. V.I. Sumin. A.V. Chernov. Volterra functional-operator equations in optimisation theory of distributed systems // in "Proceedings of International Conference "Dynamics of systems and control processes" dedicated to 90th anniversary of acad. N.N. Krasovskii. Ekaterinburg, 293-300 (2015). (in Russian). 
28. A.V. Chernov. On total global solvability of a controlled Hammerstein type equations with a varying linear operator // Vestnik Udmurt Univ. Matem. Mekh. Komputer. Nauki. 25: 2, 230-243 (2015). (in Russian).

29. A.V. Chernov. Preservation of the solvability of a semilinear global electric circuit equation // Comput. Math. Math. Phys. 58: 12, 2018-2030 (2018).

30. A.V. Chernov. The total preservation of unique global solvability of the first kind operator equation with additional controlled nonlinearity // Izv. VUZov. Matem. 11, 60-74 (2018). [Russ. Math. Iz. VUZ. 62:11, 53-66 (2018).]

31. V.A. Solonnikov. Estimates of solutions to a Navier-Stokes non-stationary system // Zap. Nauchn. Semin. Leningr. Otd. Mat. Inst. Steklova. 38, 153-231 (1973). (in Russian).

32. J. Leray. Sur le mouvement d'un liquide visqueux emplissant l'espace // Acta Math. 63: 1, 193-248 (1934).

33. A.A. Kiselev, O.A. Ladyženskaya. On the existence and uniqueness of solutions of the nonstationary problems for flows of noncompressible fluids // Izv. AN SSSR. Ser. Matem. 21: 5, 655-680 (1957). [AMS Transl. Ser.2. 24. AMS: Providence, RI, 79-106 (1963).]

34. G. Prodi. Un teorema di unicità per le equazioni di Navier-Stokes // Ann. Mat. Pura Appl. 48:4, 173-182 (1959). (in Itaian.)

35. J. Serrin. The initial value problem for the Navier-Stokes equations // in "Nonlinear Problems". Proc. Sympos. Madison. Univ. of Wisconsin Press, Madison, 69-98 (1963).

36. H. Kozono, H. Sohr. Remarks on uniqueness of weak solutions to the Navier-Stokes equations // Analysis. 16: 3, 255-271 (1996).

37. O.A. Ladyzhenskaya. Sixth problem of the millenium: Navier-Stokes equations, existence and smoothness // Uspekhi Matem. Nauk. 58: 2(350), 45-78 (2003). [Russ. Math. Surveys. 2003. 58:2, 251-286 (2003).]

38. H. Jia, V. Sverak. Are the incompressible 3d Navier-Stokes equations locally ill-posed in the natural energy space? // J. Funct. Anal. 268: 12, 3734-3766 (2015).

39. G.A. Seregin, T.N. Shilkin. Liouville-type theorems for the Navier-Stokes equations // Russ. Math. Surveys. 73:4, 661-724 (2018).

40. M.M. Karchevskii, M.F. Pavlova. Equations of mathematical physics. Additional chapters. Kazan State Univ., Kazan (2012). (in Russian).

Andrey Vladimirovich Chernov, Lobachevsky State University

of Nizhni Novgorod,

Gagarin av. 23,

603950, Nizhni Novgorod, Russia

Nizhny Novgorod State Technical

University named after R.E. Alekseev

Minin str. 24,

603950, Nizhni Novgorod, Russia

E-mail: chavnn@mail.ru 